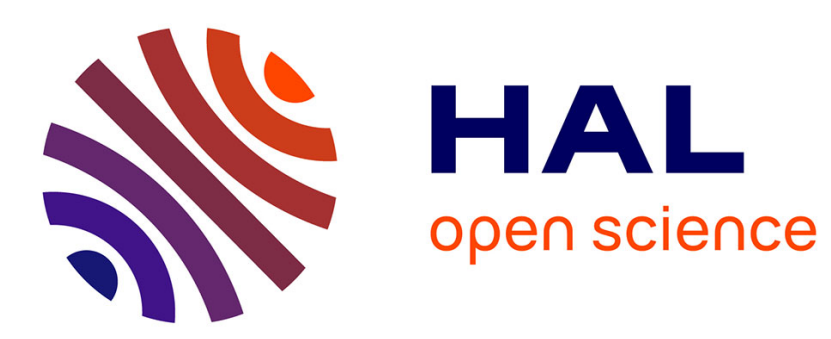

\title{
Dominant substructural vibration modes for fully-loaded spent nuclear fuel canisters
}

Olivier Ezvan, X. Zeng, R. Ghanem, B. Gencturk

\section{To cite this version:}

Olivier Ezvan, X. Zeng, R. Ghanem, B. Gencturk. Dominant substructural vibration modes for fully-loaded spent nuclear fuel canisters. Computational Mechanics, 2020, 67 (1), pp.365-384. 10.1007/s00466-020-01937-8 . hal-03245490

\section{HAL Id: hal-03245490 \\ https://hal.science/hal-03245490}

Submitted on 1 Jun 2021

HAL is a multi-disciplinary open access archive for the deposit and dissemination of scientific research documents, whether they are published or not. The documents may come from teaching and research institutions in France or abroad, or from public or private research centers.
L'archive ouverte pluridisciplinaire HAL, est destinée au dépôt et à la diffusion de documents scientifiques de niveau recherche, publiés ou non, émanant des établissements d'enseignement et de recherche français ou étrangers, des laboratoires publics ou privés. 


\title{
Dominant substructural vibration modes for fully-loaded spent nuclear fuel canisters
}

\author{
O. Ezvan · X. Zeng · R. Ghanem • B. Gencturk
}

Received: date / Accepted: date

\begin{abstract}
This paper investigates the dynamic characteristics of a multilevel structure for the transportation and storage of spent nuclear fuel (SNF) from commercial power plants. The nuclear fuel is stored in slender rods that are grouped together into fuel assemblies (FA). In a sealed cylindrical container called "canister", the FA are inserted into a honeycomb basket. The objective of this paper is to develop a computational model that accurately describes the structural dynamics of the canister, based on measurements collected from its external surface. Based on these measurements, the computational model is intended to capture the signature of the structural integrity of the internal components (FA and fuel rods). The numerous components lead to a large finite element (FE) model and numerous vibration modes and eigenfrequencies. Nevertheless, the localized connections between components enable an efficient domain decomposition with few interface coordinates. Craig-Bampton (CB) substructuring is thus used to perform the modal analysis. However, the high modal density yields a large-scale CB eigenvalue problem that necessitates a sparse solver. A block factorization by Schur complement that exploits the sparsity of the CB matrices allows an efficient calculation of the numerous eigenpairs. Many local vibration modes contribute little to the dynamics of the SNF canister. The main contribution of this paper is the development of an efficient methodology for determining the importance of each of
\end{abstract}

\section{O. Ezvan}

Laboratoire Modélisation et Simulation Multi Echelle, UMR 8208 CNRS, Université Gustave Eiffel, 5 bd Descartes, 77454 Marne-la-Vallée Cedex 02, France

E-mail: olivier.ezvan@univ-eiffel.fr

X. Zeng · R. Ghanem • B. Gencturk

Viterbi School of Engineering, University of Southern California, Los Angeles, CA, 90089, USA the substructural modes of the CB model. The removal of the least dominant substructural modes allows for reducing the CB model. Using a $75 \%$ filtering, a significant speed-up is obtained, without noticeable loss of accuracy.

Keywords Spent nuclear fuel transportation and storage, Structural dynamics, model reduction, eigenvalue problem, Craig-Bampton

\section{Introduction}

The context of this research is the nuclear safety during transportation or storage of spent nuclear fuel (SNF). The nuclear fuel pellets are assembled in long, slender metal tubes called fuel rods, which are grouped together into bundles called fuel assemblies (FA). The FA are the elements that power the reactor cores at the nuclear power plants (NPP) and are removed after depletion. FA require radiation protection and cooling for several decades. Multiple layers of packaging are required for safe transportation and interim or long-term storage. The FA are placed in a cylindrical container called the canister. A basket with honeycomb geometry is inserted into the canister to hold the FA in their respective locations. In each of the basket cells, a FA is inserted. For radiation protection, the canister is sealed and in most cases it is not meant to be opened unless it is a storage only canister and repackaging is needed for transportation. For safety, the integrity of the several levels of protection have to be assessed. The fuel rod cladding may suffer damage during and after the operation in the reactor core. Fuel rod failure is most often due to debris fretting and grid-rod fretting (the fuel rods are held by spacer grids) that initiate cracking in 
the cladding which may be worsened by hydride reorientation during the drying process. The stiffness of the FA skeleton plays an important role in grid-rod fretting. During storage, cladding oxidation and hydriding influence the fuel rod integrity. Therefore, before transportation or permanent storage, the fuel integrity has to be evaluated. Non-intrusive inspection of sealed SNF containers is the focus of recent research involving various techniques and scientific fields. This paper comes within the scope of vibration-based structural health monitoring. It consists in assessing the integrity of the internal layers or levels (the FA and the fuel rods) based on the vibration response of the external surface of the canister. For doing so, an explicit geometrical description of the several structural levels (canister, FA and fuel rods) is adopted. The ensuing finite element (FE [1]) model is large due to the large number of FA and rods, which require a finer mesh in comparison to that of the canister. In addition, the independent resonance vibrations of each of these numerous components lead to a very large number of vibration eigenmodes (or modes) and eigenfrequencies to describe the structural dynamics.

Linear vibration analysis through a FE model is usually carried out by using the vibration modes as a projection basis [2]. The dimension of the associated reduced-order model $(\mathrm{ROM})$ is in general small for a low-frequency (LF) analysis. In the LF range, the resonance peaks are well separated, whereas the high-frequency (HF) range is characterized by smooth frequency response functions (FRF) and a high modal density [3]. Other methods such as statistical energy analysis (SEA [4]) are generally preferred for the HF range, which also exhibits more variability and model uncertainty. In this research, the multilevel nature of the structure leads to a high modal density even for low frequencies. The dynamics is constituted of both long-wavelength vibrations of the canister and short-wavelength vibrations of the small components. Such a hybrid situation is typical of the medium-frequency (MF) range [3], for which neither SEA nor FE analysis prevail. Using FE analysis, the generalized eigenvalue problem (GEP) to be solved for obtaining the modal basis (the projection basis) becomes challenging. This paper presents a new methodology that tackles this difficulty inherited from the multilevel nature of the structure. In structural dynamics, the prominent eigenvalue solvers include the Shift-Invert Lanczos (SIL [5,6]) solver, the Subspace Iteration Method (SIM $[7,8]$ ), Craig-Bampton (CB [9, 10]) substructuring technique, and Automated Multilevel Substructuring (AMLS $[11,12]$ ). SIL and SIM are iterative solvers that converge to the true eigensolutions whereas CB and AMLS deliver approximate eigenso- lutions. For $\mathrm{CB}$ and AMLS, the approximation subspace is spanned by a subset of the vibration modes of the substructures, in addition to static constraint modes that ensure proper junction between the substructures. In general, CB is preferred over SIL when a large frequency band with numerous modes is considered and when accuracy is not crucial. In addition, the efficiency of SIL is lower for high-dimensional FE models, whereas in this case CB takes advantage of the divide-and-conquer paradigm. For the present case of a multilevel structure that is composed of well-separated components, CB technique is very well suited. Since the several structural levels are made up of distinct components that have limited interface, the $\mathrm{CB}$ reduction involves only a small number of static constraint modes for describing the interface forces. However, the high modal density inherited from the description of the several structural levels leads to an unusually large CB model. Usually, the CB model has a small dimension and the CB GEP that replaces the FE GEP is solved instantly for all the eigenpairs by direct solvers (based on, for instance, Householder tridiagonalization [13]). In the present case, such a direct approach is prohibitive because of this high modal density of the $\mathrm{CB}$ substructures. In [14], the CB GEP is solved by SIL. This is done efficiently by considering a block matrix factorization given by the Schur complement $[15,16]$. The sparsity of the CB matrices, which is accentuated by the small number of interface degrees of freedom (DOF) and the large number of substructural modes, is exploited. In this paper, the same strategy is employed. The focus of this paper is on the selection of a reduced set of substructural modes to decrease the dimension of the $\mathrm{CB}$ model and the resulting cost of the CB GEP. In the literature, various techniques for selecting the most important substructural modes have been proposed. The usual criterion is to consider a cutoff frequency beyond which all the modes are discarded. In $[17,18]$ the authors introduce the concept of Effective Interface Mass (EIM) that gives for any computed substructural mode its contribution relative to the complete set of substructural modes. For a given substructural mode, the EIM depends on the interface coupling matrices of the substructure but remains agnostic about the rest of the structure. In $[19,20,22]$ the importance of a substructural mode is evaluated from its contribution to the elastic forces exerted by the substructure onto its interface with the rest of the structure. The expression of this elastic force is also completely agnostic about the rest of the structure. A variant following a moment-matching approach has been proposed in [21]. In [23] the substructural modes are ranked according to their contribution to the mean kinetic and elastic 
energies over the time history, for given frequency and spatial distribution of the excitation. In [24] the ranking is defined according to the contribution to the response (rather than the energy) and requires assigning importance weights to each of the system modes (i.e., to the modes of the entire structure, as opposed to the substructural modes). In $[25,26]$ the moment-matching method of [21] is revisited and the sorting criterion is very similar to that of $[17,18]$ but multiplied by a different exponent of the substructural eigenfrequency. Finally, the moment-matching method is used in [27] for design optimization. In this paper, the importance of a substructural mode is defined through the error in decibel that the FRF of interest undergo after removal of said substructural mode. This criterion is directly related to the usage made of the CB model and does not suffer from any conceptual consideration or assumption. For computational efficiency, the modal importance is approximated through coarse frequency sampling and with a reduced set of FRF of interest, which can both be adjusted.

The paper is organized as follows. In Section 2, the usual tools and concepts for modal analysis in computational structural dynamics are presented, including SIL, CB, and domain decomposition. In Section 3, theoretical developments from paper [14] are summarized, including the efficient implementation of CB technique adapted to the high modal density exhibited by the SNF canister. Finally, in Section 4, the proposed methodology for determining the dominant substructural modes is presented.

\section{Modal analysis in computational structural dynamics}

\subsection{Direct numerical simulation}

In this paper, the structural vibrations under harmonic excitation are studied in the frequency domain. The unknown displacement field is discretized using the FE method. The resulting vector $\mathbb{U}(\omega)$ of nodal displacements is the solution of the matrix equation

$$
\left(-\omega^{2}[\mathbb{M}]+i \omega[\mathbb{D}]+[\mathbb{K}]\right) \mathbb{U}(\omega)=\mathbb{F}(\omega),
$$

in which $[\mathbb{M}],[\mathbb{D}]$, and $[\mathbb{K}]$ are the mass, damping, and stiffness FE matrices, $\mathbb{F}(\omega)$ is the vector of the external forces, and $\omega$ is the circular frequency. Equation (1) is considered for frequencies belonging to an interval (frequency band of analysis) $\mathcal{B}=2 \pi \times] 0, f_{u}$ ] with $f_{u}$ its upper bound in $\mathrm{Hz}$. The FE matrices are assumed to be positive definite and their dimension is denoted by $N$ (number of DOF). In general, the FE matrices are large and sparse. Solving Eq. (1) for many frequencies and external loads can be computationally prohibitive. Traditionally, a reduced-order model (ROM) is constructed based on the vibration eigenmodes.

\subsection{Modal analysis}

The vibration eigenmodes are the eigenvectors of the generalized eigenvalue problem (GEP)

$[\mathbb{K}] \varphi=\lambda[\mathbb{M}] \varphi$

in which $\varphi$ is an eigenmode (or mode) and $\lambda>0$ is the associated eigenvalue. The eigenfrequency in $\mathrm{Hz}$ is given by $\sqrt{\lambda} / 2 \pi$. There are $N$ finite eigenvalues but only a few $n_{e} \ll N$ of them are of interest. In general, for a vibration analysis in frequency band $\mathcal{B}$, all the modes with eigenfrequency below some $f_{c}>f_{u}$ are considered. The cutoff frequency $f_{c}$ depends for instance on the half-power bandwidth of the modes beyond $\mathcal{B}$ and is usually determined by convergence analysis. Let $[\Phi]$ denote the modal matrix that includes as columns the first $n_{e}$ modes (i.e., the modes whose eigenfrequency belongs to $\left.\left.\left.\mathcal{B}_{c}=2 \pi \times\right] 0, f_{c}\right]\right)$. The GEP can be written in matrix form as

$[\mathbb{K}][\Phi]=[\mathbb{M}][\Phi][\Lambda]$,

in which $[\Lambda]$ denotes the diagonal matrix of the $n_{e}$ eigenvalues. The modes, whose amplitude is arbitrary, satisfy orthogonality properties that, under mass normalization, are written as

$[\Phi]^{\top}[\mathbb{M}][\Phi]=\left[I_{n_{e}}\right], \quad[\Phi]^{\top}[\mathbb{K}][\Phi]=[\Lambda]$.

In Eq. (4), $\left[I_{n_{e}}\right]$ denotes the identity matrix of dimension $n_{e}$. This notation for the identity matrix is reserved throughout the article. In this paper, a modal damping model is assumed, such that the projection of the damping matrix is considered to be diagonal. It is written as $[\Phi]^{\top}[\mathbb{D}][\Phi]=2[\Xi][\Omega]$, with $[\Omega]=\sqrt{[\Lambda]}$ and where $[\Xi]$ is the diagonal matrix of the modal damping ratios. The ROM is obtained through the approximation

$\mathbb{U}(\omega)=[\Phi] \mathbf{q}(\omega)$,

where $\mathbf{q}(\omega)$ is a vector comprising $n_{e}$ generalized coordinates that are obtained by solving the diagonal matrix equation

$\left(-\omega^{2}\left[I_{n_{e}}\right]+2 \mathrm{i} \omega[\Xi][\Omega]+[\Lambda]\right) \mathbf{q}(\omega)=\mathcal{F}(\omega)$,

with $\mathcal{F}(\omega)=[\Phi]^{\top} \mathbb{F}(\omega)$. This equation is very inexpensive and can thus be solved easily for numerous frequencies and loads. The FRF $\mathbb{U}(\omega)$ need not be computed 
for every DOF of the structure. A subset of $N_{I} \mathrm{DOF}$ is considered, for which unit loads are applied and the displacements are observed. The associated FRF matrix $\left[\mathbb{U}_{I I}(\omega)\right]$ can be obtained as

$\left[\mathbb{U}_{I I}(\omega)\right]=\left[\Phi_{I}\right]\left(-\omega^{2}\left[I_{n_{e}}\right]+2 \mathrm{i} \omega[\Xi][\Omega]+[\Lambda]\right)^{-1}\left[\Phi_{I}\right]^{\top}$,

in which matrix $\left[\Phi_{I}\right]$ with dimension $\left(N_{I} \times n_{e}\right)$ is the restriction (rows) of matrix $[\Phi]$ to the $N_{I}$ DOF of interest. In this setting, the overall cost of the vibration analysis is concentrated on the eigenvalue computation of Eq. (3).

\subsection{Shift-Invert Lanczos approach}

For computing a relatively large number of modes, an efficient strategy is to divide the frequency band and use the Lanczos solver with eigenvalue shifts. This way, the cost associated with maintaining orthogonality between the eigenvectors remains linear with respect to the number of eigenvectors sought. In addition, it allows for coarse-grained parallelism. Introducing an eigenvalue shift $\sigma$, the GEP $[\mathbb{K}] \varphi=\lambda[\mathbb{M}] \varphi$ is replaced by the GEP $([\mathbb{K}]-\sigma[\mathbb{M}]) \boldsymbol{\varphi}=(\lambda-\sigma)[\mathbb{M}] \boldsymbol{\varphi}$ with both positive and negative eigenvalues. The SIL solver considers the standard eigenvalue problem $(\mathrm{SEP})([\mathbb{K}]-\sigma[\mathbb{M}])^{-1}[\mathbb{M}] \boldsymbol{\varphi}=$ $\frac{1}{\lambda-\sigma} \boldsymbol{\varphi}$ which has better eigenvalue separation. Introducing the symmetric indefinite matrix $[\mathbb{H}(\sigma)]=[\mathbb{K}]-\sigma[\mathbb{M}]$, the GEP of Eq. (2) is replaced by the SEP

$$
\left([\mathbb{H}(\sigma)]^{-1}[\mathbb{M}]\right) \boldsymbol{\varphi}=\left(\frac{1}{\lambda-\sigma}\right) \boldsymbol{\varphi}
$$

In the Lanczos algorithm, the matrix-vector product $\left([\mathbb{H}(\sigma)]^{-1}[\mathbb{M}]\right) \mathbf{x}$ is repeated through the iterations for varying $\mathbf{x}$. The ensuing linear system involving indefinite matrix $[\mathbb{H}(\sigma)]$ is usually solved with direct solvers through factorization (such as LDL [28]) rather than iterative solvers (such as conjugate gradient [29] ). In this paper, the LDL factorization of $[\mathbb{H}(\sigma)]$ is considered, such that

$$
[\mathbb{P}(\sigma)]^{\top}[\mathbb{H}(\sigma)][\mathbb{P}(\sigma)]=[\mathbb{L}(\sigma)][\mathbb{D}(\sigma)][\mathbb{L}(\sigma)]^{\top}
$$

in which $[\mathbb{L}(\sigma)]$ is a lower-triangular matrix, $[\mathbb{D}(\sigma)]$ is a block-diagonal matrix with a block size of 1 or 2 , and $[\mathbb{P}(\sigma)]$ is a permutation matrix such that $[\mathbb{P}(\sigma)]^{-1}=$ $[\mathbb{P}(\sigma)]^{\top}$. In the sequel, the dependency on $\sigma$ is omitted for simplifying the notation to

$$
[\mathbb{P}]^{\top}[\mathbb{H}][\mathbb{P}]=[\mathbb{L}][\mathbb{D}][\mathbb{L}]^{\top} .
$$

\subsection{Domain decomposition}

The sparsity of the FE matrices is related to the structural connectivity between the FE. Partitioning the structural domain $\Omega$ into $\mathcal{N}$ substructures $\Omega_{1}, \ldots, \Omega_{\mathcal{N}}$ can be beneficial for efficiency. Denoting with $B$, the DOF that belong to the boundary between substructures and with $S$, the remaining DOF that belong to the interior of the substructures, the FE matrices can be rewritten in partitioned form as

$$
[\mathbb{K}]=\left[\begin{array}{ll}
\mathbb{K}_{S S} & \mathbb{K}_{S B} \\
\mathbb{K}_{B S} & \mathbb{K}_{B B}
\end{array}\right], \quad[\mathbb{M}]=\left[\begin{array}{ll}
\mathbb{M}_{S S} & \mathbb{M}_{S B} \\
\mathbb{M}_{B S} & \mathbb{M}_{B B}
\end{array}\right]
$$

Unlike the boundary $\operatorname{DOF}(B)$, which are connected to several substructures, the substructure DOF $(S)$ are only connected to DOF belonging to the same substructure. Therefore, the submatrix $\left[\mathbb{K}_{S S}\right]$ (for instance) is block diagonal, such that

$\left[\mathbb{K}_{S S}\right]=\left[\begin{array}{cccc}\mathbb{K}_{11} & 0 & \ldots & 0 \\ 0 & \mathbb{K}_{22} & \ddots & \vdots \\ \vdots & \ddots & \ddots & 0 \\ 0 & \ldots & 0 & \mathbb{K}_{\mathcal{N N}}\end{array}\right]$

Solving the linear system $[\mathbb{K}] \mathbf{x}=\mathbf{f}$ (i.e., computing $\mathbf{x}=[\mathbb{K}]^{-1} \mathbf{f}$ ) can be performed in parallel over the substructures in conjunction with a sequential step involving the boundary, as is shown hereinafter. The following matrix decomposition of $[\mathbb{K}]$ can be shown

$\left[\begin{array}{ll}\mathbb{K}_{S S} & \mathbb{K}_{S B} \\ \mathbb{K}_{B S} & \mathbb{K}_{B B}\end{array}\right]=$

$\left[\begin{array}{cc}I_{S} & 0 \\ \mathbb{K}_{B S} \mathbb{K}_{S S}^{-1} & I_{B}\end{array}\right]\left[\begin{array}{cc}\mathbb{K}_{S S} & 0 \\ 0 & \mathbb{S}_{B B}^{\mathbb{K}}\end{array}\right]\left[\begin{array}{cc}I_{S} & \mathbb{K}_{S S}^{-1} \mathbb{K}_{S B} \\ 0 & I_{B}\end{array}\right]$

in which $\left[\mathbb{S}_{B B}^{\mathbb{K}}\right]$ is the Schur complement, such that

$\left[\mathbb{S}_{B B}^{\mathbb{K}}\right]=\left[\mathbb{K}_{B B}\right]-\left[\mathbb{K}_{B S}\right]\left[\mathbb{K}_{S S}\right]^{-1}\left[\mathbb{K}_{S B}\right]$.

Owing to Eq. (13), it can be shown (using block matrix inversion formula) that the inverse of $[\mathbb{K}]$ can be written as

$$
\begin{aligned}
& {[\mathbb{K}]^{-1}=} \\
& {\left[\begin{array}{cc}
I_{S} & -\mathbb{K}_{S S}^{-1} \mathbb{K}_{S B} \\
0 & I_{B}
\end{array}\right]\left[\begin{array}{cc}
\mathbb{K}_{S S}^{-1} & 0 \\
0 & \left(\mathbb{S}_{B B}^{\mathbb{K}}\right)^{-1}
\end{array}\right]\left[\begin{array}{cc}
I_{S} & 0 \\
-\mathbb{K}_{B S} \mathbb{K}_{S S}^{-1} & I_{B}
\end{array}\right] .}
\end{aligned}
$$

Consequently, introducing the notation $\mathbf{x}=\left(\mathbf{x}_{B}, \mathbf{x}_{S}\right)^{\top}$ and $\mathbf{f}=\left(\mathbf{f}_{B}, \mathbf{f}_{S}\right)^{\top}$, the linear system $[\mathbb{K}] \mathbf{x}=\mathbf{f}$ can be solved through the operations

$$
\begin{aligned}
& \mathbf{x}_{B}=\left[\mathbb{S}_{B B}^{\mathbb{K}}\right]^{-1}\left(\mathbf{f}_{B}-\left[\mathbb{K}_{B S}\right]\left[\mathbb{K}_{S S}\right]^{-1} \mathbf{f}_{S}\right), \\
& \mathbf{x}_{S}=\left[\mathbb{K}_{S S}\right]^{-1}\left(\mathbf{f}_{S}-\left[\mathbb{K}_{S B}\right] \mathbf{x}_{B}\right) .
\end{aligned}
$$


It can be seen in Eq. (16) that although a linear solution is required twice for the substructures (index $S$ ), it involves a block-diagonal matrix. Hence, each substructure linear system can be solved in parallel with the others. A block Cholesky [28] factorization $[\mathbb{K}]=$ $[\mathbb{U}]^{\top}[\mathbb{U}]$ can be deduced from Eq. (13) based on the Cholesky factorizations $\left[\mathbb{K}_{S S}\right]=\left[\mathbb{U}_{S}\right]^{\top}\left[\mathbb{U}_{S}\right]$ and $\left[\mathbb{S}_{B B}^{\mathbb{K}}\right]=$ $\left[\mathbb{U}_{B}\right]^{\top}\left[\mathbb{U}_{B}\right]$ (where $\left[\mathbb{U}_{S}\right]$ and $\left[\mathbb{U}_{B}\right]$ are upper-triangular matrices), by introducing the upper-triangular matrix [U] such that

$[\mathbb{U}]=\left[\begin{array}{cc}\mathbb{U}_{S} & \mathbb{U}_{S}^{-\top} \mathbb{K}_{S B} \\ 0 & \mathbb{U}_{B}\end{array}\right]$.

Similarly to Eq. (16), the Cholesky factorization $\left[\mathbb{K}_{S S}\right]=$ $\left[\mathbb{U}_{S}\right]^{\top}\left[\mathbb{U}_{S}\right]$ and the linear solution of $\left[\mathbb{U}_{S}\right]^{-\mathrm{T}}\left[\mathbb{K}_{S B}\right]$ can be performed blockwise because $\left[\mathbb{K}_{S S}\right]$ is block diagonal. Similar to Eq. (16), the linear system $[\mathbb{H}] \mathbf{x}=\mathbf{f}$ (with $[\mathbb{H}]=[\mathbb{K}]-\sigma[\mathbb{M}]$ ) can be solved according to

$\mathbf{x}_{B}=\left[\mathbb{S}_{B B}^{\mathbb{H}}\right]^{-1}\left(\mathbf{f}_{B}-\left[\mathbb{H}_{B S}\right]\left[\mathbb{H}_{S S}\right]^{-1} \mathbf{f}_{S}\right)$,

$\mathbf{x}_{S}=\left[\mathbb{H}_{S S}\right]^{-1}\left(\mathbf{f}_{S}-\left[\mathbb{H}_{S B}\right] \mathbf{x}_{B}\right)$,

in which $\left[\mathbb{S}_{B B}^{\mathbb{H}}\right]$ is the Schur complement such that $\left[\mathbb{S}_{B B}^{\mathbb{H}}\right]=$ $\left[\mathbb{H}_{B B}\right]-\left[\mathbb{H}_{B S}\right]\left[\mathbb{H}_{S S}\right]^{-1}\left[\mathbb{H}_{S B}\right]$. A block LDL factorization $[\mathbb{P}]^{\top}[\mathbb{H}][\mathbb{P}]=[\mathbb{L}][\mathbb{D}][\mathbb{L}]^{\top}$ can thus be deduced based on the LDL factorizations $\left[\mathbb{P}_{S}\right]^{\top}\left[\mathbb{H}_{S S}\right]\left[\mathbb{P}_{S}\right]=\left[\mathbb{L}_{S}\right]\left[\mathbb{D}_{S}\right]\left[\mathbb{L}_{S}\right]^{\top}$ and $\left[\mathbb{P}_{B}\right]^{\top}\left[\mathbb{S}_{B B}^{\mathbb{H}}\right]\left[\mathbb{P}_{B}\right]=\left[\mathbb{L}_{B}\right]\left[\mathbb{D}_{B}\right]\left[\mathbb{L}_{B}\right]^{\top}$ (where $\left[\mathbb{L}_{S}\right]$ and $\left[\mathbb{L}_{B}\right]$ are lower-triangular matrices, $\left[\mathbb{D}_{S}\right]$ and $\left[\mathbb{D}_{B}\right]$ are block-diagonal matrices with a block size of 1 or 2 , and $\left[\mathbb{P}_{S}\right]$ and $\left[\mathbb{P}_{B}\right]$ are permutation matrices) by introducing the lower-triangular matrix $[\mathbb{L}]$, the block-diagonal matrix $[\mathbb{D}]$ with a block size of 1 or 2 , and the permutation matrix $[\mathbb{P}]$, such that

$$
\begin{aligned}
& {[\mathbb{L}]=\left[\begin{array}{cc}
\mathbb{L}_{S} & 0 \\
\mathbb{P}_{B}^{\top} \mathbb{H}_{B S} \mathbb{H}_{S S}^{-1} \mathbb{P}_{S} \mathbb{L}_{S} & \mathbb{L}_{B}
\end{array}\right],} \\
& {[\mathbb{D}]=\left[\begin{array}{cc}
\mathbb{D}_{S} & 0 \\
0 & \mathbb{D}_{B}
\end{array}\right], \quad[\mathbb{P}]=\left[\begin{array}{cc}
\mathbb{P}_{S} & 0 \\
0 & \mathbb{P}_{B}
\end{array}\right] .}
\end{aligned}
$$

The number $\mathcal{I}([\mathbb{H}])$ of negative eigenvalues of $[\mathbb{H}]$ (inertia count $[30,31])$ is a byproduct of the above LDL factorization and it can be obtained as

$$
\begin{aligned}
\mathcal{I}([\mathbb{H}])=\mathcal{I}([\mathbb{D}]) & =\mathcal{I}\left(\left[\mathbb{D}_{B}\right]\right)+\mathcal{I}\left(\left[\mathbb{D}_{S}\right]\right) \\
& =\mathcal{I}\left(\left[\mathbb{D}_{B}\right]\right)+\sum_{J=1}^{\mathcal{N}} \mathcal{I}\left(\left[\mathbb{D}_{J}\right]\right) .
\end{aligned}
$$

The inertia count is not only useful for verifying that the eigenvalue computation by SIL did not miss any mode, it is also a way to estimate the modal density (distribution of the eigenvalues), which is necessary for splitting the frequency band and choosing the eigenvalue shifts $\sigma$.

\subsection{Craig-Bampton approach}

The CB substructuring technique provides approximate solutions to the GEP $[\mathbb{K}] \varphi=\lambda[\mathbb{M}] \varphi$ by introducing the reduced-order representation $\varphi=[V] \mathbf{q}$ with $\mathbf{q}=$ $\left(\mathbf{q}_{S}, \boldsymbol{\varphi}_{B}\right)^{\top}$, a vector of both generalized coordinates $\left(\mathbf{q}_{S}\right)$ and physical coordinates $\left(\boldsymbol{\varphi}_{B}\right)$. Following the same approach as in Section 2.2, the GEP is projected (Galerkin projection) onto the subspace spanned by the columns of matrix $[V]$, such that

$$
\left([V]^{\top}[\mathbb{K}][V]\right) \mathbf{q}=\lambda\left([V]^{\top}[\mathbb{M}][V]\right) \mathbf{q} .
$$

The reduced-order representation is given by the rectangular matrix $[V]$, such that

$$
[V]=\left[\begin{array}{cc}
\Phi_{S} & t_{S B} \\
0 & I_{B}
\end{array}\right], \quad\left[t_{S B}\right]=-\left[\mathbb{K}_{S S}\right]^{-1}\left[\mathbb{K}_{S B}\right],
$$

in which $\left[t_{S B}\right]$ is the matrix of the static constraint modes that ensure the continuity between the substructures and where the matrix $\left[\Phi_{S}\right]$ satisfies the equality $\left[\mathbb{K}_{S S}\right]\left[\Phi_{S}\right]=\left[\mathbb{M}_{S S}\right]\left[\Phi_{S}\right]\left[\Lambda_{S}\right]$. Matrices $\left[\Phi_{S}\right]$ and $\left[\Lambda_{S}\right]$ are block diagonal such that

$$
\left[\Phi_{S}\right]=\left[\begin{array}{cccc}
\Phi_{1} & 0 & \ldots & 0 \\
0 & \Phi_{2} & \ddots & \vdots \\
\vdots & \ddots & \ddots & 0 \\
0 & \ldots & 0 & \Phi_{\mathcal{N}}
\end{array}\right], \quad\left[\Lambda_{S}\right]=\left[\begin{array}{cccc}
\Lambda_{1} & 0 & \ldots & 0 \\
0 & \Lambda_{2} & \ddots & \vdots \\
\vdots & \ddots & \ddots & 0 \\
0 & \ldots & 0 & \Lambda_{\mathcal{N}}
\end{array}\right]
$$

and are obtained by solving the $\mathcal{N}$ GEP associated with the substructures:

$\left[\mathbb{K}_{J J}\right]\left[\Phi_{J}\right]=\left[\mathbb{M}_{J J}\right]\left[\Phi_{J}\right]\left[\Lambda_{J}\right], \quad J=1, \ldots, \mathcal{N}$.

For each substructure $J$, only $n_{J}$ modes out of the $N_{J}$ DOF are kept, usually by considering a cutoff frequency. In general, one has $n_{J} \ll N_{J}$ and the GEP $[K] \mathbf{q}=$ $\lambda[M] \mathbf{q}$ of Eq. (21) with $[K]=[V]^{\top}[\mathbb{K}][V]$ and $[M]=$ $[V]^{\top}[\mathbb{M}][V]$ is not very expensive. The dimension of the CB model is given by $\nu=N_{B}+\sum_{J=1}^{\mathcal{N}} n_{J}$ with $N_{B}$ the number of boundary DOF. The $\mathrm{CB}$ matrices $[K]$ and $[M]$ can be written as

$[K]=\left[\begin{array}{ll}K_{S S} & K_{B S}^{\top} \\ K_{B S} & K_{B B}\end{array}\right], \quad[M]=\left[\begin{array}{ll}M_{S S} & M_{B S}^{\top} \\ M_{B S} & M_{B B}\end{array}\right]$,

in which the submatrices are given by

$$
\begin{aligned}
{\left[K_{S S}\right]=} & {\left[\Lambda_{S}\right], \quad\left[K_{B S}\right]=[0], } \\
{\left[K_{B B}\right]=} & {\left[\mathbb{S}_{B B}^{\mathbb{K}}\right], \quad\left[M_{S S}\right]=\left[I_{S}\right], } \\
{\left[M_{B S}\right]=} & \left(\left[\mathbb{M}_{B S}\right]+\left[t_{S B}\right]^{\top}\left[\mathbb{M}_{S S}\right]\right)\left[\Phi_{S}\right], \\
{\left[M_{B B}\right]=} & {\left[\mathbb{M}_{B B}\right]+\left[\mathbb{M}_{B S}\right]\left[t_{S B}\right] } \\
& +\left(\left[\mathbb{M}_{B S}\right]\left[t_{S B}\right]\right)^{\top}+\left[t_{S B}\right]^{\top}\left[\mathbb{M}_{S S}\right]\left[t_{S B}\right] .
\end{aligned}
$$


The block structure for $[K]$ and $[M]$ is inherited from domain decomposition, as demonstrated hereinafter. The projection basis $[V]$ can be written as $[V]=[\mathbb{B}][R]$ with $[\mathbb{B}]$ and $[R]$ such that

$$
\begin{array}{rr}
{[R]=\left[\begin{array}{cc}
\Phi_{S} & 0 \\
0 & I_{B}
\end{array}\right],} & {[\mathbb{B}]=\left[\begin{array}{cc}
I_{S} & -\mathbb{K}_{S S}^{-1} \mathbb{K}_{S B} \\
0 & I_{B}
\end{array}\right],} \\
{[\mathbb{B}]^{-1}=\left[\begin{array}{cc}
I_{S} & \mathbb{K}_{S S}^{-1} \mathbb{K}_{S B} \\
0 & I_{B}
\end{array}\right] .}
\end{array}
$$

And, given the equality $[\mathbb{K}]=[\mathbb{B}]^{-\top}\left([\mathbb{B}]^{\top}[\mathbb{K}][\mathbb{B}]\right)[\mathbb{B}]^{-1}$, it can be deduced by identification from Eqs. (13) and (27) that the matrix $[\mathbb{B}]^{\top}[\mathbb{K}][\mathbb{B}]$ is block diagonal. Owing to the block structure of rectangular matrix $[R]$ (this matrix is responsible for dimension reduction), the block structure of $[K]$ and $[M]$ is thus preserved.

\section{Craig-Bampton implementation suitable for high modal density}

3.1 Craig-Bampton implementation with Shift-Invert Lanczos

In the case of high modal density (large number of modes or in this case, large number of substructural modes, $n_{J}$ ), the dimension of the CB GEP can be very high, such that the direct approach to compute all the eigenpairs of Eq. (21) (e.g., Householder tridiagonalization) is computationally prohibitive. Nevertheless, the CB matrices are rather sparse, especially in the case of low number $N_{B}$ of boundary DOF and large number $n_{S}=\sum_{J=1}^{\mathcal{N}} n_{J}$ of substructural (or component) modes. Indeed, $\left[M_{S S}\right]$ is diagonal, $\left[M_{B S}\right]$ is sparse with dense blocks, and $\left[M_{B B}\right]$ is rather dense. In this context, it is proposed that the CB GEP be solved using the SIL solver just like the usual approach for FE analysis. A large portion of the eigenpairs of the sparse GEP are sought.

As a basic tool for SIL, solving the linear system $[H] \mathbf{x}=$ $\mathbf{f}$ with $[H]=[K]-\sigma[M]$ and given vector $\mathbf{f}$ can be carried out according to

$\mathbf{x}_{B}=\left[S_{B B}^{H}\right]^{-1}\left(\mathbf{f}_{B}-\left[H_{B S}\right]\left[H_{S S}\right]^{-1} \mathbf{f}_{S}\right)$,

$\mathbf{x}_{S}=\left[H_{S S}\right]^{-1}\left(\mathbf{f}_{S}-\left[H_{S B}\right] \mathbf{x}_{B}\right)$,

in which $\left[S_{B B}^{H}\right]$ is the Schur complement such that $\left[S_{B B}^{H}\right]=$ $\left[H_{B B}\right]-\left[H_{B S}\right]\left[H_{S S}\right]^{-1}\left[H_{S B}\right]$. The submatrices $\left[H_{B B}\right]$, $\left[H_{B S}\right]$, and $\left[H_{S S}\right]$ of symmetric matrix $[H]$ are given by

$$
\begin{aligned}
& {\left[H_{B B}\right]=\left[K_{B B}\right]-\sigma\left[M_{B B}\right], \quad\left[H_{B S}\right]=-\sigma\left[M_{B S}\right]} \\
& {\left[H_{S S}\right]=\left[\Lambda_{S}\right]-\sigma\left[I_{S}\right],}
\end{aligned}
$$

from which the expression for the Schur complement $\left[S_{B B}^{H}\right]$ follows as

$$
\begin{aligned}
{\left[S_{B B}^{H}\right]=} & {\left[K_{B B}\right]-\sigma\left[M_{B B}\right] } \\
& -\sigma^{2}\left[M_{B S}\right]\left(\left[\Lambda_{S}\right]-\sigma\left[I_{S}\right]\right)^{-1}\left[M_{B S}\right]^{\top} .
\end{aligned}
$$

A block LDL factorization $[P]^{\top}[H][P]=[L][D][L]^{\top}$ can be obtained - just like in Section 2.4 - through the LDL factorizations $\left[P_{S}\right]^{\top}\left[H_{S S}\right]\left[P_{S}\right]=\left[L_{S}\right]\left[D_{S}\right]\left[L_{S}\right]^{\top}$ and $\left[P_{B}\right]^{\top}\left[S_{B B}^{H}\right]\left[P_{B}\right]=\left[L_{B}\right]\left[D_{B}\right]\left[L_{B}\right]^{\top}$ (where $\left[L_{S}\right]=\left[I_{S}\right]$ and $\left[L_{B}\right]$ are lower-triangular matrices, $\left[D_{S}\right]=\left[\Lambda_{S}\right]-$ $\sigma\left[I_{S}\right]$ and $\left[D_{B}\right]$ are block-diagonal matrices with a block size of 1 or 2 , and $\left[P_{S}\right]=\left[I_{S}\right]$ and $\left[P_{B}\right]$ are permutation matrices) by introducing the lower-triangular matrix $[L]$, the block-diagonal matrix $[D]$ with a block size of 1 or 2 , and the permutation matrix $[P]$ such that

$$
\begin{gathered}
{[L]=\left[\begin{array}{cc}
I_{S} & 0 \\
-\sigma P_{B}^{\top} M_{B S}\left(\Lambda_{S}-\sigma I_{S}\right)^{-1} & L_{B}
\end{array}\right],} \\
{[D]=\left[\begin{array}{cc}
\Lambda_{S}-\sigma I_{S} & 0 \\
0 & D_{B}
\end{array}\right], \quad[P]=\left[\begin{array}{cc}
I_{S} & 0 \\
0 & P_{B}
\end{array}\right] .}
\end{gathered}
$$

The inertia count $\mathcal{I}([H])$ is a byproduct of the above LDL factorization, given by

$$
\begin{array}{r}
\mathcal{I}([H])=\mathcal{I}([D])=\mathcal{I}\left(\left[D_{B}\right]\right)+\mathcal{I}\left(\left[\Lambda_{S}\right]-\sigma\left[I_{S}\right]\right) \\
=\mathcal{I}\left(\left[D_{B}\right]\right)+\sum_{J=1}^{\mathcal{N}} \mathcal{I}\left(\left[\Lambda_{J}\right]-\sigma\left[I_{J}\right]\right) .
\end{array}
$$

3.2 Craig-Bampton model for the fully-loaded spent nuclear fuel canister

In this paper, we are interested in performing the vibration analysis of a fully-loaded SNF canister (FLSNFC). The canister is a cylindrical container with thick upper and lower circular plates inside which a basket with honeycomb structure is placed (see Fig.1). This basket has 68 cells inside each of which a FA is inserted. A FA is constituted of a stiff frame that holds together close to a hundred nuclear fuel rods that are stacked together along their length (see Fig.2). The FLSNFC clearly exhibits three structural levels that correspond to the FLSNFC components: the lower level is constituted of the fuel rods, the medium level of 68 identical FA, and the upper level of the canister and its basket. The structural connection between the components is not continuous but rather discrete and consequently, it is possible to define an advantageous domain decomposition that entails a small number of boundary DOF, namely $N_{B}=1140$ with the $68 \mathrm{FA}$ and the main frame 


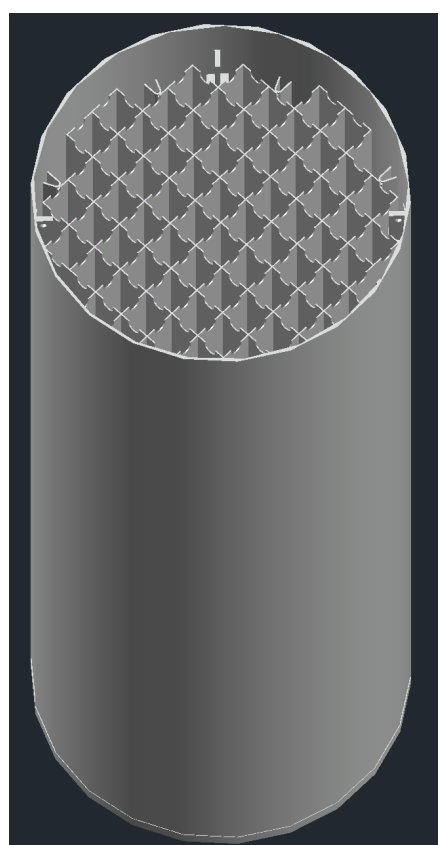

Fig. 1 Frame substructure (canister + basket), represented without the thick canister lid.

(canister and basket) as substructures $(\mathcal{N}=69)$. There are only $N_{\beta}=27$ boundary DOF for each FA. On the other hand, the slender fuel rods, which are numerous, are responsible for the presence of a very large number of modes. Nearly half a million modes will be considered for the vibration analysis. In addition, due to the fine mesh necessary for representing the lower scales, the FE model has $N=N_{B}+68 \times N_{A}+N_{F}=$ $1140+68 \times 1,935,837+2,209,084=133,847,140 \mathrm{DOF}$ (with $N_{A}$ the number of DOF for each FA and $N_{F}$ the number of DOF for the frame substructure). A CB model is implemented according to Section 3.1. The FE matrices $\left[\mathbb{K}^{F}\right]$ and $\left[\mathbb{M}^{F}\right]$ for the frame substructure, which include interior (substructure) DOF denoted by $F$ and boundary DOF denoted by $B$, can be written as $\left[\mathbb{K}^{F}\right]=\left[\begin{array}{ll}\mathbb{K}_{F F} & \mathbb{K}_{F B} \\ \mathbb{K}_{B F} & \mathbb{K}_{B B}^{F}\end{array}\right], \quad\left[\mathbb{M}^{F}\right]=\left[\begin{array}{ll}\mathbb{M}_{F F} & \mathbb{M}_{F B} \\ \mathbb{M}_{B F} & \mathbb{M}_{B B}^{F}\end{array}\right]$

For the frame, there are $N_{B}=1140$ static constraint modes to compute according to $\left[t_{F B}\right]=-\left[\mathbb{K}_{F F}\right]^{-1}\left[\mathbb{K}_{F B}\right]$. In addition, there is a given number $n_{F}$ of frame modes to compute from the GEP

$\left[\mathbb{K}_{F F}\right]\left[\Phi_{F}\right]=\left[\mathbb{M}_{F F}\right]\left[\Phi_{F}\right]\left[\Lambda_{F}\right]$.

This number $n_{F}$ of modes is determined by the cutoff frequency, which depends on the accuracy desired. Introducing the matrix $\left[V_{F}\right]$ such that

$\left[V_{F}\right]=\left[\begin{array}{cc}\Phi_{F} & t_{F B} \\ 0 & I_{B}\end{array}\right]$
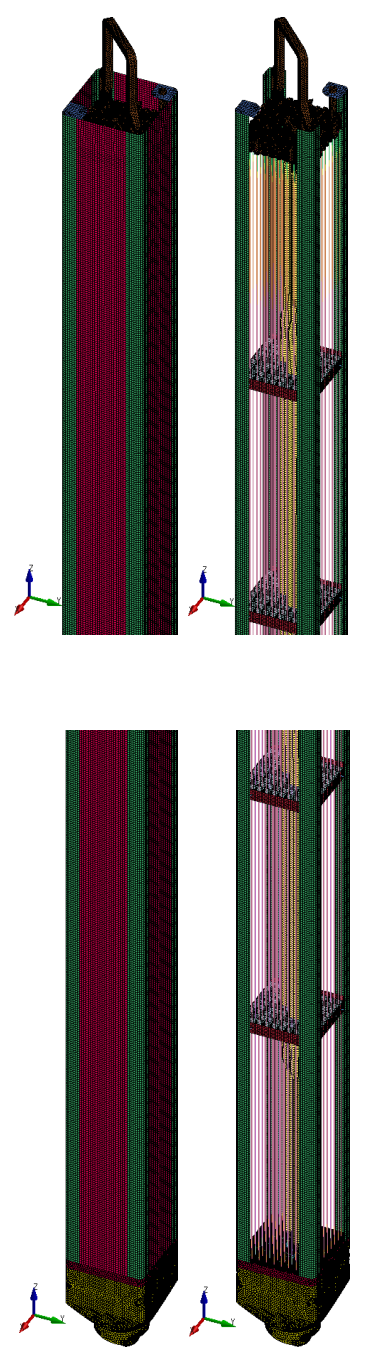

Fig. 2 Fuel assembly (partial views with and without surrounding channel).

the $\mathrm{CB}$ matrices $\left[K^{F}\right]=\left[V_{F}\right]^{T}\left[\mathbb{K}^{F}\right]\left[V_{F}\right]$ and $\left[M^{F}\right]=$ $\left[V_{F}\right]^{T}\left[\mathbb{M}^{F}\right]\left[V_{F}\right]$ associated with the frame substructure are obtained based on their submatrices $\left[K_{F F}\right]=\left[\Lambda_{F}\right]$, $\left[K_{B F}\right]=[0],\left[M_{F F}\right]=\left[I_{F}\right]$, and

$$
\begin{aligned}
{\left[K_{B B}^{F}\right]=} & {\left[\mathbb{K}_{B B}^{F}\right]+\left[\mathbb{K}_{B F}\right]\left[t_{F B}\right] } \\
{\left[M_{B B}^{F}\right]=} & {\left[\mathbb{M}_{B B}^{F}\right]+\left[\mathbb{M}_{B F}\right]\left[t_{F B}\right]+\left(\left[\mathbb{M}_{B F}\right]\left[t_{F B}\right]\right)^{\top} } \\
& +\left[t_{F B}\right]^{\top}\left[\mathbb{M}_{F F}\right]\left[t_{F B}\right] \\
{\left[M_{B F}\right]=} & \left(\left[\mathbb{M}_{B F}\right]+\left[t_{F B}\right]^{\top}\left[\mathbb{M}_{F F}\right]\right)\left[\Phi_{F}\right] .
\end{aligned}
$$

For the CB model developed for the FLSNFC, the Schur complement $\left[S_{B B}^{H}\right]$ of Eq. (30) is given by the contributions from the frame and from the $68 \mathrm{FA}$. The contribution $\left[S_{B B}^{H, F}\right]$ from the frame is given by

$$
\begin{aligned}
{\left[S_{B B}^{H, F}\right]=} & {\left[K_{B B}^{F}\right]-\sigma\left[M_{B B}^{F}\right] } \\
& -\sigma^{2}\left[M_{B F}\right]\left(\left[\Lambda_{F}\right]-\sigma\left[I_{F}\right]\right)^{-1}\left[M_{B F}\right]^{\top} .
\end{aligned}
$$


The FE matrices $\left[\mathbb{K}^{A}\right]$ and $\left[\mathbb{M}^{A}\right]$ for one FA (the $68 \mathrm{FA}$ are all identical), which include interior (substructure) DOF denoted by $A$ and boundary DOF denoted by $\beta$, can be written as

$\left[\mathbb{K}^{A}\right]=\left[\begin{array}{ll}\mathbb{K}_{A A} & \mathbb{K}_{A \beta} \\ \mathbb{K}_{\beta A} & \mathbb{K}_{\beta \beta}^{A}\end{array}\right], \quad\left[\mathbb{M}^{A}\right]=\left[\begin{array}{ll}\mathbb{M}_{A A} & \mathbb{M}_{A \beta} \\ \mathbb{M}_{\beta A} & \mathbb{M}_{\beta \beta}^{A}\end{array}\right]$

For the FA, there are $N_{\beta}=27$ static constraint modes to compute according to $\left[t_{A \beta}\right]=-\left[\mathbb{K}_{A A}\right]^{-1}\left[\mathbb{K}_{A \beta}\right]$. In addition, there is a given number $n_{A}$ of frame modes to compute from the GEP

$\left[\mathbb{K}_{A A}\right]\left[\Phi_{A}\right]=\left[\mathbb{M}_{A A}\right]\left[\Phi_{A}\right]\left[\Lambda_{A}\right]$.

This number $n_{A}$ of modes is determined by the cutoff frequency, which depends on the accuracy desired. Introducing the matrix $\left[V_{A}\right]$ such that

$\left[V_{A}\right]=\left[\begin{array}{cc}\Phi_{A} & t_{A \beta} \\ 0 & I_{\beta}\end{array}\right]$

the $\mathrm{CB}$ matrices $\left[K^{A}\right]=\left[V_{A}\right]^{T}\left[\mathbb{K}^{A}\right]\left[V_{A}\right]$ and $\left[M^{A}\right]=$ $\left[V_{A}\right]^{T}\left[\mathbb{M}^{A}\right]\left[V_{A}\right]$ associated with the FA substructure are obtained based on their submatrices $\left[K_{A A}\right]=\left[\Lambda_{A}\right]$, $\left[K_{\beta A}\right]=[0],\left[M_{A A}\right]=\left[I_{A}\right]$ and

$\left[K_{\beta \beta}^{A}\right]=\left[\mathbb{K}_{\beta \beta}^{A}\right]+\left[\mathbb{K}_{\beta A}\right]\left[t_{A \beta}\right]$,

$\left[M_{\beta \beta}^{A}\right]=\left[\mathbb{M}_{\beta \beta}^{A}\right]+\left[\mathbb{M}_{\beta A}\right]\left[t_{A \beta}\right]$

$$
+\left(\left[\mathbb{M}_{\beta A}\right]\left[t_{A \beta}\right]\right)^{\top}+\left[t_{A \beta}\right]^{\top}\left[\mathbb{M}_{A A}\right]\left[t_{A \beta}\right],
$$

$\left[M_{\beta A}\right]=\left(\left[\mathbb{M}_{\beta A}\right]+\left[t_{A \beta}\right]^{\top}\left[\mathbb{M}_{A A}\right)\left[\Phi_{A}\right]\right.$.

The contribution $\left[S_{\beta \beta}^{H, A}\right]$ from one FA is given by

$$
\begin{aligned}
{\left[S_{\beta \beta}^{H, A}\right]=} & {\left[K_{\beta \beta}^{A}\right]-\sigma\left[M_{\beta \beta}^{A}\right] } \\
& -\sigma^{2}\left[M_{\beta A}\right]\left(\left[\Lambda_{A}\right]-\sigma\left[I_{A}\right]\right)^{-1}\left[M_{\beta A}\right]^{\top} .
\end{aligned}
$$

It can be deduced from Eq. (30) that the Schur complement for the FLSNFC CB model is given by

$\left[S_{B B}^{H}\right]=\sum_{J=1}^{\mathcal{N}}\left[S_{B B}^{H, J}\right]$,

in which, for $J=1,\left[S_{B B}^{H, J}\right]=\left[S_{B B}^{H, F}\right]$ and, for $J>1$, the nonzero terms of $\left[S_{B B}^{H, J}\right]$ are given by $\left[S_{\beta \beta}^{H, A}\right]$. In this paper, we are only interested in the structural vibrations as can be obtained from the external surface of the canister (which is sealed for radiation protection), for the purpose of inverse identification of the damage to the internal components (lower scales). Consequently, the $N_{I}$ DOF of interest associated with FRF matrix $\left[\mathbb{U}_{I I}(\omega)\right]$ belong to the frame. To solve Eq. (7), only $\left[\Phi_{I}\right]$ and $[\Lambda]$ need to be computed, with the columns $\varphi_{I}$ of $\left[\Phi_{I}\right]$ hence given by

$\boldsymbol{\varphi}_{I}=\left[\Phi_{F}^{I}\right] \mathbf{q}_{F}+\left[t_{F B}^{I}\right] \boldsymbol{\varphi}_{B}$,

where $\left[\Phi_{F}^{I}\right]$ and $\left[t_{F B}^{I}\right]$ denote the restrictions of the matrices $\left[\Phi_{F}\right]$ and $\left[t_{F B}\right]$ to the DOF of interest.

\subsection{Error quantification}

Partial error measure For quantifying the error due to the $\mathrm{CB}$ approximation, the approximate FRF may be compared with the exact FRF obtained with the exact modes. Of course, the exact modes are not available and it is the purpose of the CB approach to avoid having to compute them. The strategy proposed is to compare the FRF associated with (modal) superposition of a reduced set of $n_{d} \ll n_{e}$ modes, rather than the true FRF (obtained upon convergence with a large number $n_{e}$ of modes). In other words, the error quantification is carried out by comparing the FRF restricted to a subspace spanned by $n_{d}$ modes. Or, by using as quantity of interest the contribution of $n_{d}$ given modes. The $n_{d}$ exact modes are computed by SIL solver with domain decomposition (see Section 2.4) and the value of $n_{d}$ is chosen so that the computation is affordable. The use of domain decomposition allows the advantageous structural connectivity to be leveraged.

Generic error measure Let $U_{i j}(\omega)$ denote one entry of the reference FRF matrix $\left[\mathbb{U}_{I I}(\omega)\right]$ of Eq. (7) (obtained, for instance, through exact modes). Let $\widetilde{U}_{i j}(\omega)$ denote the corresponding approximate FRF (obtained, for instance, through the CB model). Introducing the moduli in decibel $(\mathrm{dB})$ scale $u_{i j}(\omega)=20 \log _{10}\left|U_{i j}(\omega)\right|$ and $\widetilde{u}_{i j}(\omega)=20 \log _{10}\left|\widetilde{U}_{i j}(\omega)\right|$, the corresponding error measure $\epsilon_{i j}$ in decibel is defined as

$\epsilon_{i j}=\sqrt{\frac{1}{|\mathcal{B}|} \int_{\mathcal{B}}\left(u_{i j}(\omega)-\widetilde{u}_{i j}(\omega)\right)^{2} d \omega}$,

in which $|\mathcal{B}|$ is the length of the frequency band of analysis. Then, the global error measure related to all the FRF is defined as

$$
\begin{aligned}
\epsilon=\mu_{\epsilon}+k_{\epsilon} \sigma_{\epsilon}, \quad \mu_{\epsilon} & =\frac{1}{N_{I}^{2}} \sum_{i=1}^{N_{I}} \sum_{j=1}^{N_{I}} \epsilon_{i j}, \quad k_{\epsilon}>0, \\
\sigma_{\epsilon}^{2} & =\frac{1}{N_{I}^{2}-1} \sum_{i=1}^{N_{I}} \sum_{j=1}^{N_{J}}\left(\epsilon_{i j}-\mu_{\epsilon}\right)^{2}
\end{aligned}
$$

in which $k_{\epsilon}$ is a parameter that controls the confidence level associated with the decibel error $\epsilon$.

Error quantification for the FLSNFC The FLSNFC exhibits many vibration modes that are due to the isolated vibrations of the small-scale components. These local modes (see Fig. 3 right), as opposed to global modes (see Fig. 3 left) that involve an in-phase deformation of the entire structure, do not necessarily contribute much to the structural response. In fact, the 


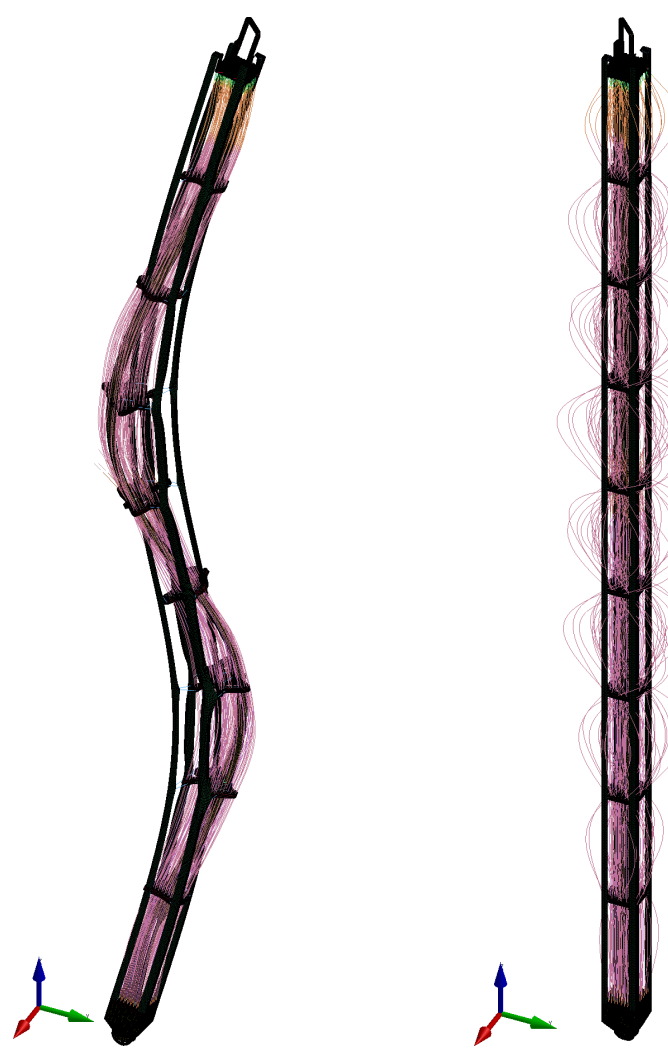

Fig. 3 Example of global mode (left) and local mode (right) for the fuel assembly.

majority of them have a negligible impact on the FRF. For better robustness of the error quantification, it is proposed that the $n_{d}$ modes be chosen as the most dominant ones, that is to say the modes that contribute the most to the FRF. To determine which are the dominant modes, a modal importance criterion is defined based on the error $\epsilon$ obtained through mode removal. That is, the importance of number- $\alpha$ vibration mode $\varphi_{\alpha}$ is given by the error $\epsilon$ that is obtained when using all the $n_{e}$ modes at the exception of mode $\varphi_{\alpha}$ for computing the FRF matrix.

The procedure is as follows. The $n_{e}$ vibration modes are approximated by the $\mathrm{CB}$ model. The list of the $n_{d}$ dominant modes is determined by calculating the modal importance of the $n_{e}$ approximate CB modes (one $\mathrm{CB}$ mode is removed at a time and the resulting FRF is compared to the reference FRF with all the $n_{e} \mathrm{CB}$ modes). These dominant modes are then exactly computed using SIL with frequency slices obtained with eigenvalue shifts corresponding to the approximate $\mathrm{CB}$ eigenvalues. For the error quantification, having correspondence (same rank) between the CB eigenvalues and the exact eigenvalues is crucial. Otherwise, it is not the same modes that are compared and the resulting error quantification is not related to the $\mathrm{CB}$ approximation.

\section{Reduced-order model based on dominant substructural modes}

Due to the presence of numerous local modes, the CB eigenvalue problem $[K] \mathbf{q}=\lambda[M] \mathbf{q}$ of Eq. (21) remains quite computationally intensive despite the CB implementation suitable for high modal density described in [14] and summarized in Section 3. In this paper, a methodology and an algorithm are developed to decrease the cost of this computation. The objective is not to compute the $n_{e} \mathrm{CB}$ modes (which provide an approximation to the reduced basis $[\Phi]$ ) but instead, to compute a reduced basis that can differ from $[\Phi]$ or its $\mathrm{CB}$ approximation. It is intended that the associated ROM gives accurate FRF. To achieve the cost reduction, the strategy is to reduce the number of substructural modes used in the reduced basis $[V]$ of the $\mathrm{CB}$ model (i.e., decrease $\left.n_{S}=\sum_{J=1}^{\mathcal{N}} n_{J}\right)$. In order to only keep dominant substructural modes, the same procedure as in Section 3.3 is used. That is, one substructural (or component) mode is removed at a time and its dominance or importance is defined as the resulting error $\epsilon$ defined in Eq. (46). To apply this strategy, a reference FRF must be available. It is recalled that the error we are interested in here is that relative to the CB model and that we want to avoid solving the $\mathrm{CB}$ eigenvalue problem. It is proposed to consider as reference FRF, the FRF obtained with the $\mathrm{CB}$ model through direct numerical simulation. For doing so, it is necessary to replace the modal damping model with another damping model, because the modes are not available. It is proposed to use a hysteretic damping model, which coincides with the modal damping model at the eigenfrequencies with appropriate frequency-dependent loss factor $\eta(\omega)=2 \xi(\omega)$ (one must have: $\forall \alpha=1, \ldots, n_{e} \quad \xi\left(\omega_{\alpha}\right)=$ $\xi_{\alpha}$, with $\omega_{\alpha}$ the eigenfrequency of mode $\alpha$ and $\xi_{\alpha}$ as its modal damping ratio). The $\mathrm{CB}$ matrices are given by

$$
\begin{array}{r}
{[K]=\left[\begin{array}{cc}
\Lambda_{S} & 0 \\
0 & K_{B B}
\end{array}\right], \quad[M]=\left[\begin{array}{cc}
I_{S} & M_{B S}^{\top} \\
M_{B S} & M_{B B}
\end{array}\right],} \\
{[D]=\frac{2 \xi(\omega)}{\omega}[K] .}
\end{array}
$$

The CB dynamic stiffness matrix $[H(\omega)]=-\omega^{2}[M]+$ $\mathrm{i} \omega[D]+[K]$ is given by:

$$
\begin{aligned}
& {[H(\omega)]=\left[\begin{array}{l}
H_{S S}(\omega) \\
H_{B S}(\omega)
\end{array} H_{B S}^{\top}(\omega)\right.} \\
& {\left[H_{S S}(\omega)\right]=-\omega^{2}\left[I_{S}\right]+(1+2 i \xi(\omega))\left[\Lambda_{S}\right]} \\
& {\left[H_{B S}(\omega)\right]=-\omega^{2}\left[M_{B S}\right]} \\
& {\left[H_{B B}(\omega)\right]=-\omega^{2}\left[M_{B B}\right]+(1+2 i \xi(\omega))\left[K_{B B}\right] .}
\end{aligned}
$$

The CB representation $\boldsymbol{\varphi}=[V] \mathbf{q}$ for the modes translates to $\mathbb{U}(\omega)=[V] \mathbf{q}(\omega)$ for the FRF (letters $\mathbf{q}$ and 
$\mathbf{q}$ differ $)$, with $\mathbb{U}(\omega)=\left(\mathbb{U}_{S}(\omega), \mathbb{U}_{B}(\omega)\right)^{T}$ and $\mathbf{q}(\omega)=$ $\left(\mathbf{q}_{S}(\omega), \mathbf{q}_{B}(\omega)\right)^{T}$. The FRF of the frame (where the DOF of interest are located) is obtained through

$$
\begin{aligned}
& \mathbb{U}(\omega)=[V] \mathbf{q}(\omega) \\
\Rightarrow & \mathbb{U}_{S}(\omega)=\left[\Phi_{S}\right] \mathbf{q}_{S}(\omega)+\left[t_{S B}\right] \mathbf{q}_{B}(\omega) \\
\Rightarrow & \mathbb{U}_{F}(\omega)=\left[\Phi_{F}\right] \mathbf{q}_{F}(\omega)+\left[t_{F B}\right] \mathbf{q}_{B}(\omega) .
\end{aligned}
$$

Similar to Section 2.4, the unknown vectors $\mathbf{q}_{F}(\omega)$ and $\mathbf{q}_{B}(\omega)$ can be obtained using the Schur complement. Let $\left[S_{B B}^{H}(\omega)\right]$ denote the Schur complement such that

$\left[S_{B B}^{H}(\omega)\right]=$

$\left[H_{B B}(\omega)\right]-\left[H_{B S}(\omega)\right]\left[H_{S S}(\omega)\right]^{-1}\left[H_{B S}(\omega)\right]^{\top}$.

Notation $\left[S_{B B}^{H}(\omega)\right]$ should not be confused with notation $\left[S_{B B}^{H}\right]$ (and $[H(\omega)]$ with $[H]$, etc.). The matrix $[H]$ defined in Section 3.1 does not include the damping term and does not depend on frequency (it depends on the eigenvalue shift $\sigma$ ). Based on frequencydependent Schur complement $\left[S_{B B}^{H}(\omega)\right]$, the unknown vectors $\mathbf{q}_{B}(\omega)$ and $\mathbf{q}_{F}(\omega)$ of Eq. (49) under application of an external load $\mathbb{F}(\omega)$ can be obtained as

$$
\begin{aligned}
& \mathbf{q}_{B}(\omega)= \\
& {\left[S_{B B}^{H}(\omega)\right]^{-1}\left(\mathbf{f}_{B}(\omega)-\left[H_{B F}(\omega)\right]\left[H_{F F}(\omega)\right]^{-1} \mathbf{f}_{F}(\omega)\right),} \\
& \mathbf{q}_{F}(\omega)=\left[H_{F F}(\omega)\right]^{-1}\left(\mathbf{f}_{F}(\omega)-\left[H_{B F}(\omega)\right]^{\top} \mathbf{q}_{B}(\omega)\right),
\end{aligned}
$$

in which $\mathbf{f}_{B}(\omega)$ and $\mathbf{f}_{F}(\omega)$ are the subvectors of the vector $\mathbf{f}(\omega)=\left(\mathbf{f}_{S}(\omega), \mathbf{f}_{B}(\omega)\right)^{T}$ given by $\mathbf{f}(\omega)=[V]^{T} \mathbb{F}(\omega)$. From Eqs. (49) and (51), it can be deduced that

$$
\begin{aligned}
\mathbb{U}_{F}(\omega)= & {\left[\Phi_{F}\right]\left[H_{F F}(\omega)\right]^{-1} \mathbf{f}_{F}(\omega) } \\
& +\left(\left[t_{F B}\right]-\left[\Phi_{F}\right]\left[H_{F F}(\omega)\right]^{-1}\left[H_{B F}\right]^{\top}\right) \mathbf{q}_{B}(\omega)
\end{aligned}
$$

For the case of unit point loads associated with the FRF matrix $\left[\mathbb{U}_{I I}(\omega)\right]$ of Eq. $(7)$, one has $\mathbf{f}_{F}(\omega)=\left[\Phi_{F}^{I}\right]^{\top}$ and $\mathbf{f}_{B}(\omega)=\left[t_{F B}^{I}\right]^{\top}$, which yields

$\left[\mathbb{U}_{I I}(\omega)\right]=\left[L_{I I}(\omega)\right]+\left[L_{I B}(\omega)\right] \mathbf{q}_{B}^{I}(\omega)$,

in which

$$
\begin{aligned}
{\left[L_{I I}(\omega)\right] } & =\left[\Phi_{F}^{I}\right]\left[H_{F F}(\omega)\right]^{-1}\left[\Phi_{F}^{I}\right]^{\top} \\
{\left[L_{I B}(\omega)\right] } & =\left[t_{F B}^{I}\right]-\left[\Phi_{F}^{I}\right]\left[H_{F F}(\omega)\right]^{-1}\left[H_{B F}\right]^{\top} \\
& =\left[t_{F B}^{I}\right]+\omega^{2}\left[\Phi_{F}^{I}\right]\left[H_{F F}(\omega)\right]^{-1}\left[M_{B F}\right]^{\top} \\
\mathbf{q}_{B}^{I}(\omega) & =\left[S_{B B}^{H}(\omega)\right]^{-1}\left[L_{I B}(\omega)\right]^{\top} .
\end{aligned}
$$

Similarly to Eq. (43), the Schur complement $\left[S_{B B}^{H}(\omega)\right]$ of Eq. (50) is given by

$$
\begin{aligned}
{\left[S_{B B}^{H}(\omega)\right]=} & {\left[H_{B B}(\omega)\right]-\left[H_{B F}(\omega)\right]\left[H_{F F}(\omega)\right]^{-1}\left[H_{B F}(\omega)\right]^{\top} } \\
& -\sum_{J=2}^{69}\left[H_{B J}(\omega)\right]\left[H_{J J}(\omega)\right]^{-1}\left[H_{B J}(\omega)\right]^{\top}, \quad(55)
\end{aligned}
$$

in which for all $J=2, \ldots, 69$ one has $\left[H_{J J}(\omega)\right]=$ $\left[H_{A A}(\omega)\right]=-\omega^{2}\left[I_{A}\right]+(1+2 i \xi(\omega))\left[\Lambda_{A}\right]$ and where the nonzero terms of $\left[H_{B J}(\omega)\right]$ are given by $\left[H_{\beta A}(\omega)\right]=$ $-\omega^{2}\left[M_{\beta A}\right]$. It follows that

$$
\begin{aligned}
{\left[S_{B B}^{H}(\omega)\right]=} & {\left[H_{B B}(\omega)\right] } \\
& -\omega^{4}\left(\left[s_{B B}^{H, F}(\omega)\right]+\sum_{J=2}^{69}\left[s_{B B}^{H, J}(\omega)\right]\right),
\end{aligned}
$$

with $\left[s_{B B}^{H, F}(\omega)\right]=\left[M_{B F}\right]\left[H_{F F}(\omega)\right]^{-1}\left[M_{B F}\right]^{\top}$ and where the nonzero terms of $\left[s_{B B}^{H, J}(\omega)\right]$ are given by $\left[s_{\beta \beta}^{H, A}(\omega)\right]=$ $\left[M_{\beta A}\right]\left[H_{A A}(\omega)\right]^{-1}\left[M_{\beta A}\right]^{\top}$, for all $J=2, \ldots, 69$.

From Eqs. (53) and (54), one gets

$$
\left[\mathbb{U}_{I I}(\omega)\right]=\left[L_{I I}(\omega)\right]+\left[L_{I B}(\omega)\right]\left[S_{B B}^{H}(\omega)\right]^{-1}\left[L_{I B}(\omega)\right]^{\top} .
$$

Let $\left[\widetilde{\mathbb{U}}_{I I}(\omega)\right]$ denote the approximation of $\left[\mathbb{U}_{I I}(\omega)\right]$ resulting from the removal of a single mode of a given FA. Since neither $\left[L_{I I}(\omega)\right]$ nor $\left[L_{I B}(\omega)\right]$ depend on the set of FA modes retained, only the Schur complement $\left[S_{B B}^{H}(\omega)\right]$ is affected by the removal of a FA mode. Thus, let $\left[\widetilde{S}_{B B}^{H}(\omega)\right]$ denote the approximation of $\left[S_{B B}^{H}(\omega)\right]$ resulting from the mode removal. In the expression of $\left[S_{B B}^{H}(\omega)\right]$ in Eq. (56), the only term that is affected by the removal of a FA mode is $\left[s_{B B}^{H, J}(\omega)\right]$, which is defined through $\left[s_{\beta \beta}^{H, A}(\omega)\right]$. Thus, let $\left[\widetilde{s}_{\beta \beta}^{H, A}(\omega)\right]$ denote the approximation of $\left[s_{\beta \beta}^{H, A}(\omega)\right]$ resulting from the mode removal. Let $\left[\widetilde{M}_{\beta A}\right]$ be the matrix obtained by removing from matrix $\left[M_{\beta A}\right]$ the column corresponding to the removed FA mode, and let $\left[\widetilde{H}_{A A}(\omega)\right]$ be the diagonal matrix obtained by removing from $\left[H_{A A}(\omega)\right]$ the row and column corresponding to the removed FA mode. Due to the outer product form of $\left[s_{\beta \beta}^{H, A}(\omega)\right]$, it can be deduced that

$$
\left[\widetilde{s}_{\beta \beta}^{H, A}(\omega)\right]=\left[s_{\beta \beta}^{H, A}(\omega)\right]-\frac{1}{h(\omega)} \mathbf{v}_{\beta} \mathbf{v}_{\beta}^{\top}
$$

in which $\mathbf{v}_{\beta}$ denotes the column vector that is removed from $\left[M_{\beta A}\right]$ to obtain $\left[\widetilde{M}_{\beta A}\right]$ and where the scalar function $h(\omega)$ is given by $h(\omega)=-\omega^{2}+(1+2 i \xi(\omega)) \lambda_{A}$, 
with $\lambda_{A}$ the eigenvalue of the removed FA mode. Replacing $\left[s_{\beta \beta}^{H, A}(\omega)\right]$ with $\left[\widetilde{s}_{\beta \beta}^{H, A}(\omega)\right]$ for the relevant $\mathrm{FA}$, the modified Schur complement $\left[\widetilde{S}_{B B}^{H}(\omega)\right]$ becomes

$$
\begin{gathered}
{\left[\widetilde{S}_{B B}^{H}(\omega)\right]=\left[H_{B B}(\omega)\right]} \\
-\omega^{4}\left(\left[s_{B B}^{H, F}(\omega)\right]+\left(\sum_{J=2}^{69}\left[s_{B B}^{H, J}(\omega)\right]\right)-\frac{1}{h(\omega)} \mathbf{v}_{B} \mathbf{v}_{B}^{\top}\right) \\
=\left[S_{B B}^{H}(\omega)\right]+\frac{w^{4}}{h(\omega)} \mathbf{v}_{B} \mathbf{v}_{B}^{\top},
\end{gathered}
$$

in which $\mathbf{v}_{B}$ is a vector with dimension $N_{B}$ whose nonzeros elements are given by vector $\mathbf{v}_{\beta}$. It can be seen from Eq. (59) that $\left[\widetilde{S}_{B B}^{H}(\omega)\right]$ is a rank-1 modification of $\left[S_{B B}^{H}(\omega)\right]$. For given square matrix $A$ and matrices $U, C$, and $V$ with compatible dimensions, ShermanMorrison-Woodbury formula [28] is written as

$$
\begin{aligned}
& (A+U C V)^{-1}= \\
& A^{-1}-A^{-1} U\left(C^{-1}+V A^{-1} U\right)^{-1} V A^{-1} .
\end{aligned}
$$

Replacing $A$ with $\left[S_{B B}^{H}(\omega)\right], U$ with $\mathbf{v}_{B}, V$ with $\mathbf{v}_{B}^{\top}$, and $C$ with $\frac{w^{4}}{h(\omega)}$ yields

$$
\begin{gathered}
{\left[\widetilde{S}_{B B}^{H}(\omega)\right]^{-1}=\left[S_{B B}^{H}(\omega)\right]^{-1}-\left[S_{B B}^{H}(\omega)\right]^{-1} \mathbf{v}_{B} \ldots} \\
\times\left(\frac{h(\omega)}{\omega^{4}}+\mathbf{v}_{B}^{\top}\left[S_{B B}^{H}(\omega)\right]^{-1} \mathbf{v}_{B}\right)^{-1} \mathbf{v}_{B}^{\top}\left[S_{B B}^{H}(\omega)\right]^{-1} .
\end{gathered}
$$

Introducing the vector function $\mathbf{p}_{B}(\omega)=\left[S_{B B}^{H}(\omega)\right]^{-1} \mathbf{v}_{B}$ and the scalar function $a(\omega)=\frac{h(\omega)}{\omega^{4}}+\mathbf{v}_{B}^{\top} \mathbf{p}_{B}(\omega)$, the inverse of the modified Schur complement $\left[\widetilde{S}_{B B}^{H}(\omega)\right]$ can be written as

$$
\left[\widetilde{S}_{B B}^{H}(\omega)\right]^{-1}=\left[S_{B B}^{H}(\omega)\right]^{-1}-\frac{1}{a(\omega)} \mathbf{p}_{B}(\omega) \mathbf{p}_{B}^{\top}(\omega) .
$$

It can be deduced that the FRF matrix $\left[\widetilde{U}_{I I}(\omega)\right]$ resulting from the removal of a single mode of a given FA can be written as

$$
\begin{aligned}
{\left[\widetilde{\mathbb{U}}_{I I}(\omega)\right] } & =\left[L_{I I}(\omega)\right]+\left[L_{I B}(\omega)\right]\left[\widetilde{S}_{B B}^{H}(\omega)\right]^{-1}\left[L_{I B}(\omega)\right]^{\top} \\
& =\left[L_{I I}(\omega)\right]+\left[L_{I B}(\omega)\right] \ldots \\
\times & \left(\left[S_{B B}^{H}(\omega)\right]^{-1}-\frac{1}{a(\omega)} \mathbf{p}_{B}(\omega) \mathbf{p}_{B}^{\top}(\omega)\right)\left[L_{I B}(\omega)\right]^{\top} \\
& =\left[\mathbb{U}_{I I}(\omega)\right]-\frac{1}{a(\omega)} \mathbf{q}_{I}(\omega) \mathbf{q}_{I}^{\top}(\omega)
\end{aligned}
$$

in which the complex vector $\mathbf{q}_{I}(\omega)$ is defined as $\mathbf{q}_{I}(\omega)=$ $\left[L_{I B}(\omega)\right] \mathbf{p}_{B}(\omega)$. The dimension $N_{I}$ of the FRF matrix $\left[\mathbb{U}_{I I}(\omega)\right]$ as well as the frequency sampling can be adjusted to decrease the cost for computing $\left[\mathbb{U}_{I I}(\omega)\right]$ and $\left[\widetilde{U}_{I I}(\omega)\right]$ through Eqs. (57) and (63).
Summary of the operations The first step is to compute the exact response $\left[\mathbb{U}_{I I}(\omega)\right]$ defined in Eq. (57), for a given frequency sampling and $N_{I}$ given DOF of interest. For this, it is assumed that the following matrices have been computed: $\left[K_{B B}\right],\left[M_{B B}\right],\left[t_{F B}^{I}\right],\left[\Phi_{F}^{I}\right]$, $\left[M_{B F}\right]$, and $\left[M_{\beta A}\right]$.

For all sampling frequency $\omega$, compute (loop over $\omega$ ):

- $\left[H_{F F}(\omega)\right]=(1+2 i \xi(\omega))\left[\Lambda_{F}\right]-\omega^{2}\left[I_{F}\right]$

- $\left[H_{A A}(\omega)\right]=(1+2 i \xi(\omega))\left[\Lambda_{A}\right]-\omega^{2}\left[I_{A}\right]$

- $\left[P_{B F}(\omega)\right]=\left[M_{B F}\right]\left[H_{F F}(\omega)\right]^{-1}$

This consists of a real dense $\left(N_{B} \times n_{F}\right)\left(n_{F} \times n_{F}\right)$ complex diagonal matrix product.

- $\left[P_{I F}(\omega)\right]=\left[\Phi_{F}^{I}\right]\left[H_{F F}(\omega)\right]^{-1}$

This consists of a real dense $\left(N_{I} \times n_{F}\right)\left(n_{F} \times n_{F}\right)$ complex diagonal matrix product.

- $\left[L_{I B}(\omega)\right]=\left[t_{F B}^{I}\right]+\omega^{2}\left[\Phi_{F}^{I}\right]\left[P_{B F}(\omega)\right]^{\top}$

This is a real dense $\left(N_{I} \times n_{F}\right)\left(n_{F} \times N_{B}\right)$ complex dense matrix product.

- $\left[L_{I I}(\omega)\right]=\left[P_{I F}(\omega)\right]\left[\Phi_{F}^{I}\right]^{\top}$

This is a complex dense $\left(N_{I} \times n_{F}\right)\left(n_{F} \times N_{I}\right)$ complex dense matrix product.

- $\left[s_{B B}^{H, F}(\omega)\right]=\left[P_{B F}(\omega)\right]\left[M_{B F}\right]^{\top}$

This is a complex dense $\left(N_{B} \times n_{F}\right)\left(n_{F} \times N_{B}\right)$ real dense matrix product.

- $\left[s_{\beta \beta}^{H, A}(\omega)\right]=\left[M_{\beta A}\right]\left[H_{A A}(\omega)\right]^{-1}\left[M_{\beta A}\right]^{\top}$

This consists of a real dense $\left(N_{\beta} \times n_{A}\right)\left(n_{A} \times n_{A}\right)$ complex diagonal matrix product and of a complex dense $\left(N_{\beta} \times n_{A}\right)\left(n_{A} \times N_{\beta}\right)$ real dense matrix product.

- $\left[H_{B B}(\omega)\right]=-\omega^{2}\left[M_{B B}\right]+(1+2 i \xi(\omega))\left[K_{B B}\right]$

- $\left[S_{B B}^{H}(\omega)\right]=\left[H_{B B}(\omega)\right]$

$$
-\omega^{4}\left(\left[s_{B B}^{H, F}(\omega)\right]+\sum_{J=2}^{69}\left[s_{B B}^{H, J}(\omega)\right]\right)
$$

- LDL factorization of $\left[S_{B B}^{H}(\omega)\right]$

This matrix is complex dense $\left(N_{B} \times N_{B}\right)$.

- $\left[P_{B I}(\omega)\right]=\left[S_{B B}^{H}(\omega)\right]^{-1}\left[L_{I B}(\omega)\right]^{\top}$

This is a complex dense $\left(N_{B} \times N_{B}\right)\left(N_{B} \times N_{I}\right)$ complex dense linear solve.

- $\left[\mathbb{U}_{I I}(\omega)\right]=\left[L_{I I}(\omega)\right]+\left[P_{B I}(\omega)\right]^{\top}\left[L_{I B}(\omega)\right]^{\top}$

This entails a complex dense $\left(N_{I} \times N_{B}\right)\left(N_{B} \times N_{I}\right)$ complex dense matrix product.

- $\left[u_{I I}(\omega)\right]=20 \log _{10}\left|\left[\mathbb{U}_{I I}(\omega)\right]\right| \quad$ where $\log _{10}$ and modulus $|$.$| are applied term by term.$ 
For each sampling frequency $\omega$, the following matrices are saved to disk:

- the LDL factorization of complex dense $\left(N_{B} \times N_{B}\right)$ matrix $\left[S_{B B}^{H}(\omega)\right]$

- complex dense $\left(N_{B} \times N_{I}\right)$ matrix $\left[P_{B I}(\omega)\right]$

- complex dense $\left(N_{I} \times N_{I}\right)$ matrix $\left[\mathbb{U}_{I I}(\omega)\right]$

- real dense $\left(N_{I} \times N_{I}\right)$ matrix $\left[u_{I I}(\omega)\right]$

The next step is to compute the error induced by removing the FA modes one at a time. Each FA can be dealt with in parallel of the others. Although the FA are identical and consequently, share the same modes, their contributions to the dynamics of the FLSNFC is in general different, due to the different locations of the FA. A loop over the 68 FA is considered thereafter. Typically, one compute node can be assigned to each FA. For each FA, matrices $\left[M_{\beta A}\right]$ and $\left[\Lambda_{A}\right]$ are loaded in memory. In addition, denoting as $n_{\omega}$ the number of frequency points, an $\left(n_{\omega} \times n_{A} \times N_{I} \times N_{I}\right)$ array $\left[E_{I I}^{\alpha}(\omega)\right]$ is initialized to obtain the FRF error for each frequency and associated with each mode $\alpha=1, \ldots, n_{A}$ of the FA. The FRF error for each frequency is given by the integrand in Eq. (45).

Then, for all sampling frequency $\omega$, do (loop over $\omega)$ :

- load the LDL factorization of the complex dense $\left(N_{B} \times N_{B}\right)$ matrix $\left[S_{B B}^{H}(\omega)\right]$

- load the complex dense $\left(N_{B} \times N_{I}\right)$ matrix $\left[P_{B I}(\omega)\right]$

- load the complex dense $\left(N_{I} \times N_{I}\right)$ matrix $\left[\mathbb{U}_{I I}(\omega)\right]$

- load the real dense $\left(N_{I} \times N_{I}\right)$ matrix $\left[u_{I I}(\omega)\right]$

- For each mode $\alpha=1, \ldots, n_{A}$ do (loop over $\alpha$ ):

- extract vector $\mathbf{v}_{\beta}$ from matrix $\left[M_{\beta A}\right]$ to obtain vector $\mathbf{v}_{B}$

- compute $\mathbf{p}_{B}(\omega)=\left[S_{B B}^{H}(\omega)\right]^{-1} \mathbf{v}_{B}$

This is a complex dense $\left(N_{B} \times N_{B}\right)\left(N_{B} \times 1\right)$ real sparse linear solve.

- compute $h(\omega)=-\omega^{2}+(1+2 \mathrm{i} \xi(\omega)) \lambda_{A}$

- compute $a(\omega)=\frac{h(\omega)}{\omega^{4}}+\mathbf{v}_{B}^{\top} \mathbf{p}_{B}(\omega)$

This involves a real sparse $\left(1 \times N_{B}\right)\left(N_{B} \times N_{1}\right)$ complex dense dot product.

- compute $\mathbf{q}_{I}(\omega)=\left[P_{B I}(\omega)\right]^{\top} \mathbf{v}_{B}$

This is a complex dense $\left(N_{I} \times N_{B}\right)\left(N_{B} \times 1\right)$ real sparse matrix-vector product.
- compute $\left[\widetilde{U}_{I I}(\omega)\right]=\left[\mathbb{U}_{I I}(\omega)\right]-\frac{1}{a(\omega)} \mathbf{q}_{I}(\omega) \mathbf{q}_{I}^{\top}(\omega)$ This is a complex dense $\left(N_{I} \times 1\right)\left(1 \times N_{I}\right)$ complex dense outer product.

- For all $i=1, \ldots, N_{I}$ and $j=1, \ldots, N_{I}$, compute the integrand $e_{i j}=\left(u_{i j}(\omega)-\widetilde{u}_{i j}(\omega)\right)^{2}$ in Eq. (45). Then, store these $N_{I}^{2}$ values in array $\left[E_{I I}^{\alpha}(\omega)\right]$.

After the nested loops over $\omega$ and $\alpha$ are done, for a given $\mathrm{FA}$, the array $\left[E_{I I}^{\alpha}(\omega)\right]$ is filled up. For each mode $\alpha=1, \ldots, n_{A}$ of a given FA, the error measure $\epsilon_{i j}$ from Eq. (45) is computed for given $i \in\left\{1, \ldots, N_{I}\right\}$ and $j \in\left\{1, \ldots, N_{I}\right\}$ based on $n_{\omega}$ components of $\left[E_{I I}^{\alpha}(\omega)\right]$. For each mode $\alpha=1, \ldots, n_{A}$ of a given FA, the error measure $\epsilon$ from Eq. (46) is then computed based on the $N_{I}^{2}$ error measures $\epsilon_{i j}$. For each mode of each FA, its importance measure is given by its error measure $\epsilon$. The FA modes are then ranked according to this importance measure and a truncation is considered. Applying this truncation leads to a CB model that is incomplete, in the sense that not all the substructural modes below the original cutoff frequency are kept. The absence of numerous substructural modes leads to the filtering of numerous system modes (as opposed to substructural modes) through the GEP of Eq. (21) of the incomplete CB model. Thereby, not only the CB GEP of Eq. (21) is less expensive, but the system modes obtained are fewer and still constitute an adequate projection basis to represent the vibration response (the FRF). Thus, a reduced set of $n \leq n_{e}$ dominant vibration modes that accurately describes the structural dynamics is obtained at a proportionally reduced cost. It should be noted that in general, these dominant "modes" do not correspond neither to the modes solution of Eq. (2) nor to those obtained after solution of Eq. (21) with the complete (standard) CB model.

\section{Numerical results}

\subsection{Multiscale modal analysis}

In this section, some of the results presented in [14] are repeated for clarity and completeness.

It is recalled that the frame (canister + basket) has $N_{F}=2,209,084$ DOF and that the FA has $N_{A}=$ $1,935,837$ DOF. Performing a domain decomposition with the frame $(J=1)$ and each of the $68 \mathrm{FA}(J=$ $2, \ldots, 69)$ as substructures, there are only $N_{B}=1140$ boundary DOF. The FE model has $N=N_{B}+N_{F}+$ $68 \times N_{A}=133,847,140$ DOF. The objective is to compute the FRF matrix $\left[\mathbb{U}_{I I}(\omega)\right]$ of Eq. (7) gathering all 
the combinations of FRF from the $N_{I}=1581$ DOF of interest whose locations are depicted in Fig. 4. Such a

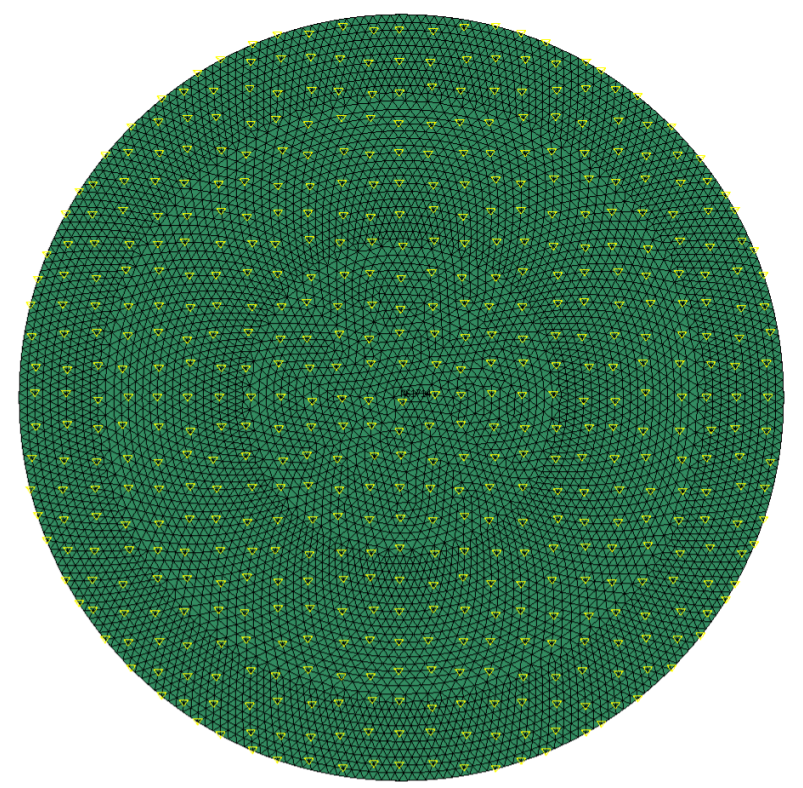

Fig. 4 Location of the $N_{I}=1581$ DOF of interest on the bottom plate of the canister.

fine observation grid is postulated to be needed for an inverse identification of damage to the internal components. Concerning the boundary conditions, the FLSNFC is suspended from the top as shown in Fig. 5. The FLSNFC is fixed at these two attachments and rigid body motion is prevented. The $N_{I}$ DOF of interest belong to the outer surface of the canister bottom plate, as depicted in Fig. 6. The FA rest in a vertical position within their respective basket cells. The vibrations of the FA are transmitted to the bottom plate through a slender nose piece but are not directly transmitted to the thick top lid. It should be noted that the basket is not connected to the cylindrical shell of the canister. In addition, the FA are not connected to the walls of their basket cell except at the top through height springs that secure their horizontal positioning. The methodology described in Section 3.2 is applied using a cluster of compute nodes, which all have roughly the same computational power. The GEP of Eq. (34) is solved for the first $n_{F}=211,057$ modes by LSDYNA software [33] using SIL, in $\sim 60 \mathrm{~h}$ with 180 nodes. The $N_{B}=1140$ static constraint modes $\left[t_{F B}\right]$ are computed by MATLAB software [34] in $\sim 3.5 \mathrm{~h}$ with one node. The GEP of Eq. (39) is solved for the first $n_{A}=46,383$ modes by LS-DYNA software using SIL, in $\sim 5 \mathrm{~h}$ with 180 nodes. The $N_{\beta}=27$ static constraint modes $\left[t_{A \beta}\right]$ are computed by MATLAB software in $\sim 15 \mathrm{~min}$ with one node. In this paper, all the re-

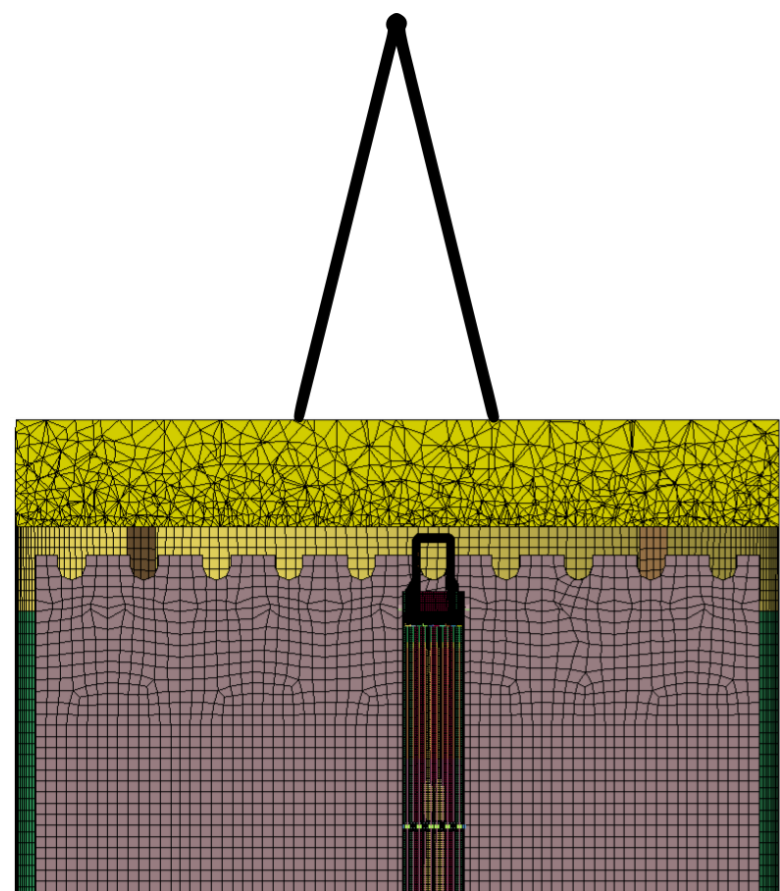

Fig. 5 Top of a longitudinal mid-section of the canister loaded with one fuel assembly (the whole details of the basket are not represented). The structure is fixed at the top of the thick canister lid through two attachments.

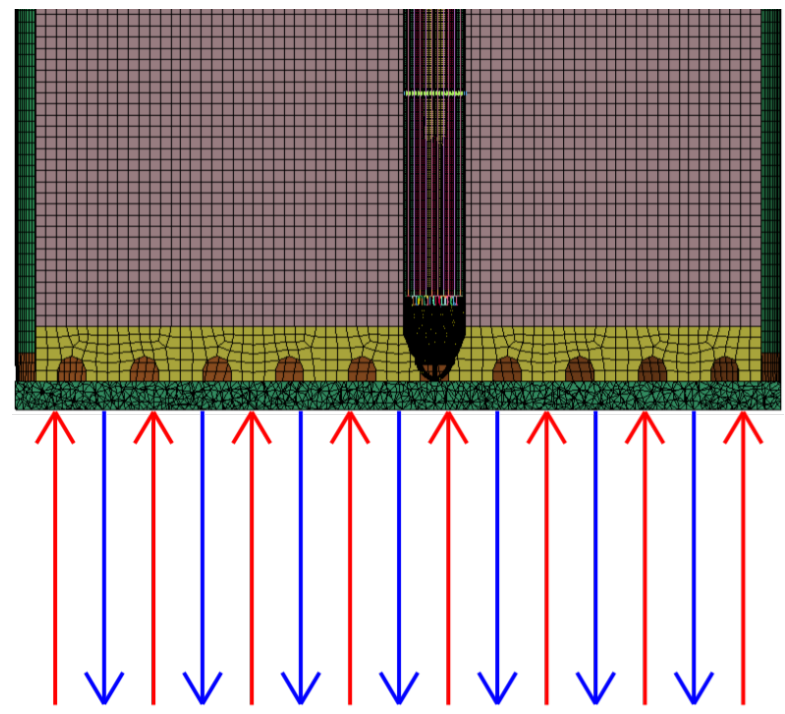

Fig. 6 Bottom of a longitudinal mid-section of the canister loaded with one fuel assembly (the whole details of the basket are not represented). The external loads and dynamic response monitoring are restricted to the outer surface of the canister bottom plate.

maining calculations are done with MATLAB software. The values for $n_{F}$ and $n_{A}$ correspond to a truncation to $20 \mathrm{kHz}$ frequency. The objective of the CB model is to calculate all the modes up to $f_{c}=1200 \mathrm{~Hz}$. The very high frequency truncation at $20 \mathrm{kHz}$ is used to achieve an almost exact solution as will be shown and 
relaxed later in this paper. This cutoff frequency explains the large values for $n_{F}$ and $n_{A}$ and associated high computational cost. Using this $\mathrm{CB}$ model of dimension $\nu=N_{B}+n_{F}+68 \times n_{A}=3,366,241$, the CB GEP of Eq. (21) is solved for the first $n_{e}=458,910$ modes found below $f_{c}$, in $\sim 3.8 \mathrm{~h}$ with 50 nodes. For error quantification (see Section 3.3), the $n_{e}=458,910$ $\mathrm{CB}$ modes are removed one at a time and the error $\epsilon$ of Eq. (46) is evaluated with $k_{\epsilon}=4$ and in considering a reduced set of $N_{I}=63 \mathrm{DOF}$ of interest for increased efficiency (see Fig. 7). The greater the error,

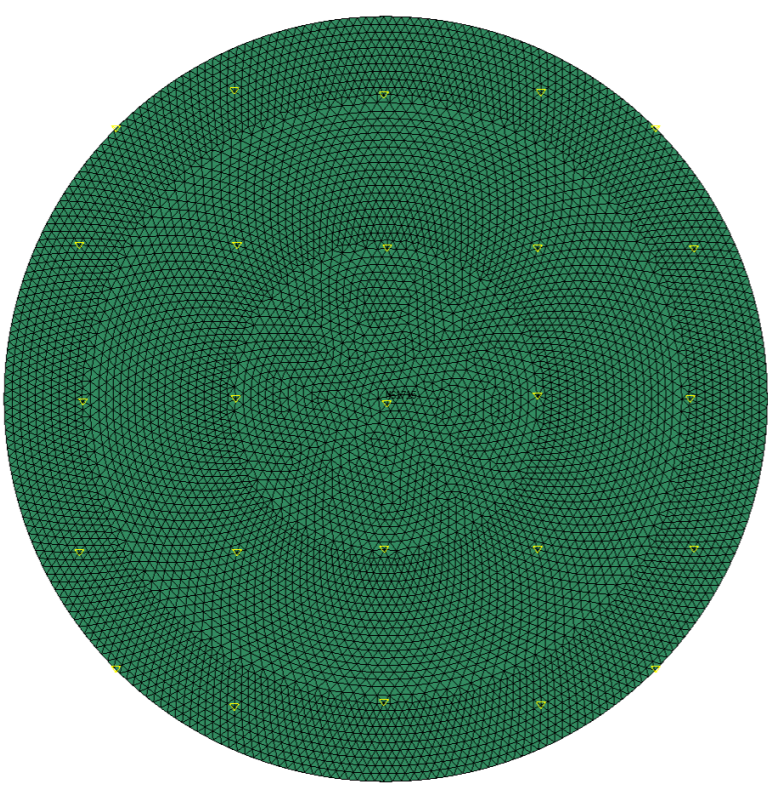

Fig. 7 Location of the $N_{I}=63$ DOF of interest considered for defining the dominant modes.

the greater the modal importance. The CB modes are sorted according to their importance (or dominance). The $n_{d}=200$ most dominant CB modes are considered for accuracy verification. Based on the eigenvalues of the $n_{d}=200$ most dominant CB modes, the corresponding 200 modes are recomputed with no approximation using SIL solver (exact modes): for each of the $n_{d}=200$ modes, an eigenvalue shift $\sigma$ is introduced, and only the closest mode is computed. Although the advantageous structural connectivity between components is leveraged by the domain decomposition linear solver presented in Section 2.4, the $n_{d}=200$ exact modes are computed in $\sim 35 \mathrm{~h}$ with 25 nodes.

The FRF matrix restricted to these $n_{d}=200$ modes is computed up to $f_{u}=1000 \mathrm{~Hz}$ based on the $\mathrm{CB}$ modes on the one hand and based on the exact modes on the other hand. The associated error measures of Eq. (46) are evaluated. Figure 8 depicts the distribution of error $\epsilon_{i j}$ of Eq. (46) associated with the FRF matrix of the $N_{I}=1581$ DOF of interest depicted in Fig. 4. One

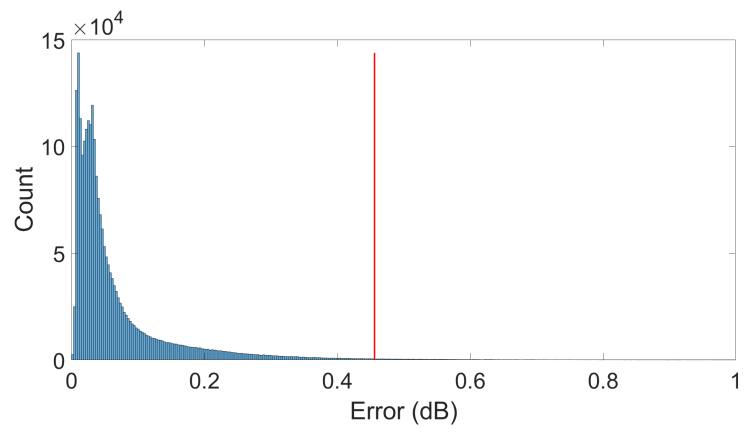

Fig. 8 Distribution of the error $\epsilon_{i j}$ for the $20 \mathrm{kHz}$ CraigBampton model with respect to the exact modes, both restricted to the $n_{d}=200$ most dominant modes. Red line: global error $\epsilon=\mu_{\epsilon}+4 \times \sigma_{\epsilon}=0.46 \mathrm{~dB}$.

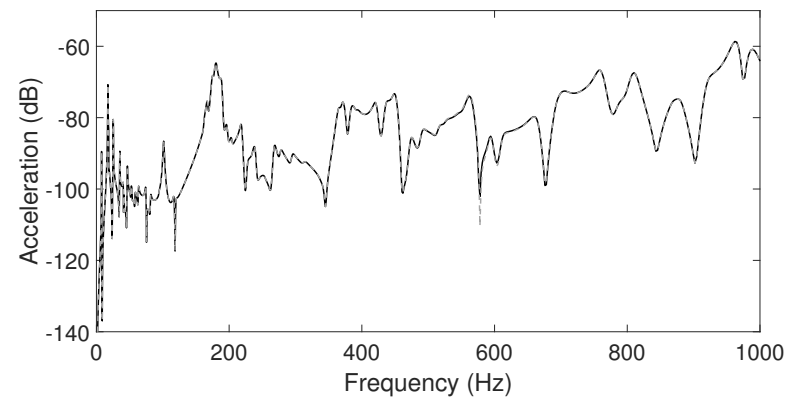

Fig. 9 FRF comparison between the $20 \mathrm{kHz}$ Craig-Bampton model (dashed gray) and the exact model (black), both restricted to the $n_{d}=200$ most dominant modes. Error: $\epsilon_{i j}=0.46 \mathrm{~dB}$.

FRF for which the error is representative of the global error $\epsilon$ is plotted in Fig. 9, based on which it can be deduced that the $20 \mathrm{kHz} \mathrm{CB}$ model is very accurate (it should be noted that roughly $99 \%$ of the FRF have an even smaller error, as can be seen in Fig. 8). Now that it has been verified that the $\mathrm{CB}$ model with $20 \mathrm{kHz}$ truncation is accurate, it can be used as a reference. Such a high cutoff frequency was necessary to ensure the $\mathrm{CB}$ modes and the exact modes have corresponding ranks. The objective of this work is to define an efficient methodology for obtaining an accurate model for the vibration analysis through the bottom plate using the $N_{I}$ DOF of the FRF matrix. This methodology is then to be used for various cases of damage or uncertainty introduced to the internal components. It is assumed that the frame substructure is not subject to damage or uncertainty and consequently, its modal matrix $\left[\Phi_{F}\right]$ and static constraint modes $\left[t_{F B}\right]$ need not be recomputed. With respect to the reference $\mathrm{CB}$ model with $20 \mathrm{kHz}$ 
truncation, several approximations are now introduced for the purpose of increased efficiency.

A convergence analysis of the $\mathrm{CB}$ model with respect to the cutoff frequency is carried out. The CB model global error $\epsilon$ is calculated for multiple truncations and the convergence curve is plotted in Fig. 10. It is seen that the error converges to zero and that a $20 \mathrm{kHz}$ truncation is clearly unnecessary. For a cutoff frequency of $4 \mathrm{kHz}$, the global error is $\epsilon=0.38 \mathrm{~dB}$, which is lower than the error of the reference $\mathrm{CB}$ model with $20 \mathrm{kHz}$ truncation (partial error with respect to the exact modes). For the

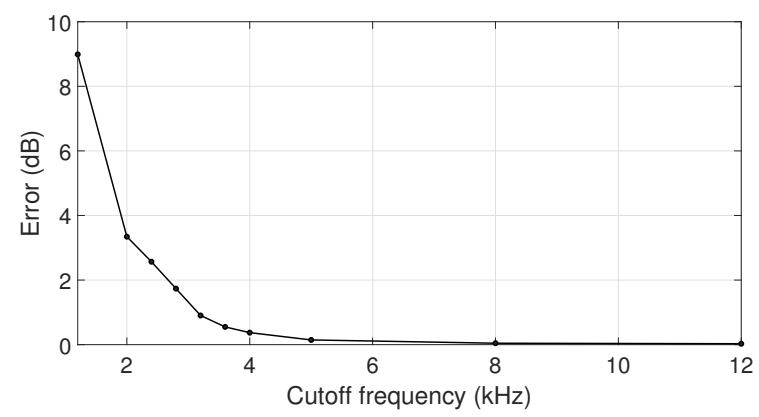

Fig. 10 Convergence of the Craig-Bampton model error $\epsilon=$ $\mu_{\epsilon}+4 \times \sigma_{\epsilon}$ with respect to the cutoff frequency.

$\mathrm{CB}$ model with $4 \mathrm{kHz}$ truncation, the distribution of the FRF error $\epsilon_{i j}$ (for all the FRF of the $N_{I}=1581$ DOF of interest) is plotted in Fig. 11. Using the $4 \mathrm{kHz} \mathrm{CB}$

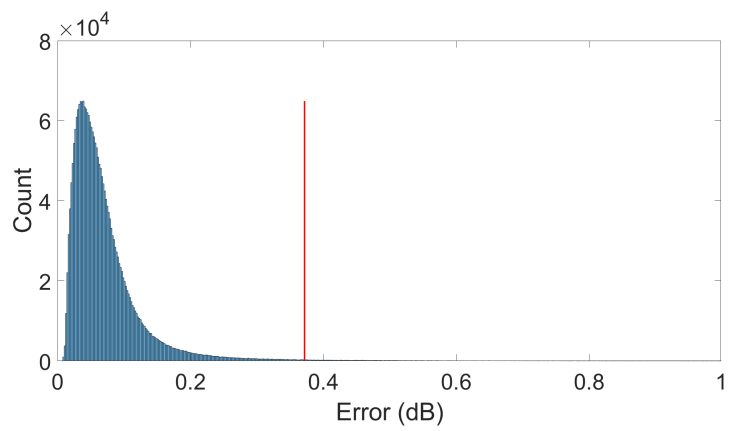

Fig. 11 Distribution of the $4 \mathrm{kHz}$ Craig-Bampton model error $\epsilon_{i j}$. Global error $\epsilon=\mu_{\epsilon}+4 \times \sigma_{\epsilon}=0.38 \mathrm{~dB}$ (red line).

model, one FRF for which the error is representative of the global error $\epsilon=0.38 \mathrm{~dB}$ is computed and plotted in Fig. 12, based on which it can be deduced that the $4 \mathrm{kHz}$ $\mathrm{CB}$ model does not lose significant accuracy. The $4 \mathrm{kHz}$ truncation is therefore selected. For this truncation, one has $n_{F}=16,623$ and $n_{A}=14,064$, which yields a CB model with dimension $\nu=974,115$. The corresponding CB GEP is solved for the first $n_{e}=458,910$ modes in $\sim 118$ min with 68 nodes. Since damage or uncertainty

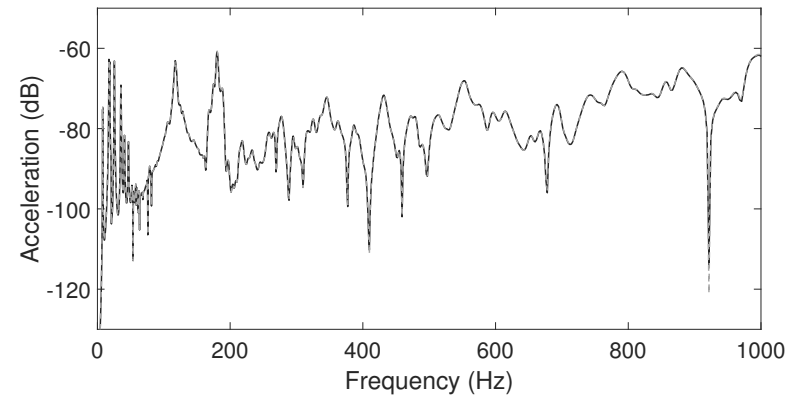

Fig. 12 FRF comparison between the $4 \mathrm{kHz}$ Craig-Bampton model (dashed gray) and the $20 \mathrm{kHz}$ Craig-Bampton reference (black). Error: $\epsilon_{i j}=0.38 \mathrm{~dB}$.

are to be introduced to the internal components (to the 68 FA), the FA eigenvalue computation of Eq. (39) has to be repeated many times. Since the FA is also a multilevel structure with localized connections between the fuel rods and their supports, a CB model is also introduced for the FA. This $\mathrm{CB}$ model is referred to as "inner CB" and the previous CB model is now referred to as "outer CB". These are nested CB models (see [14] for details). The domain decomposition associated with the inner $\mathrm{CB}$ is presented in Fig. 13. It is composed of, from left to right and top to bottom: the channel, the two water rods (for neutron moderation), the upper tie plate, the $9 \times 92$ fuel rod segments (there are 92 fuel rods), the eight spacer grids (that hold the fuel rods together), the lower tie plate, and the extremity of the bottom nose piece. A convergence analysis is carried out with respect to the cutoff frequency for the inner CB. It is found that an inner truncation of $6 \mathrm{kHz}$ is sufficient to retain the same level of accuracy, as demonstrated by Figs. 14 and 15, which respectively show the distribution of the error and one example of FRF comparison that has the same error as the global error (i.e., equal to $\epsilon=0.41 \mathrm{~dB}$ ). The FA CB GEP is solved for all the modes below $4 \mathrm{kHz}$ in $\sim 6$ min with 1 node. As a conclusion, using 68 nodes, it takes about $118+6=124$ minutes to perform the multiscale modal analysis, without significant loss of accuracy.

\subsection{Proposed reduced-order model}

As a further cost reduction step, the methodology proposed in Section 4 is now applied. It first requires the FA modes to be sorted in accordance with their contribution to the FRF matrix. The $n_{A}=14,047 \mathrm{FA}$ modes that have been computed by $6 \mathrm{kHz}$ inner $\mathrm{CB}$ are removed one at a time to determine their importance (or dominance). More precisely, the algorithm presented in Section 4 is implemented and executed with 68 nodes 

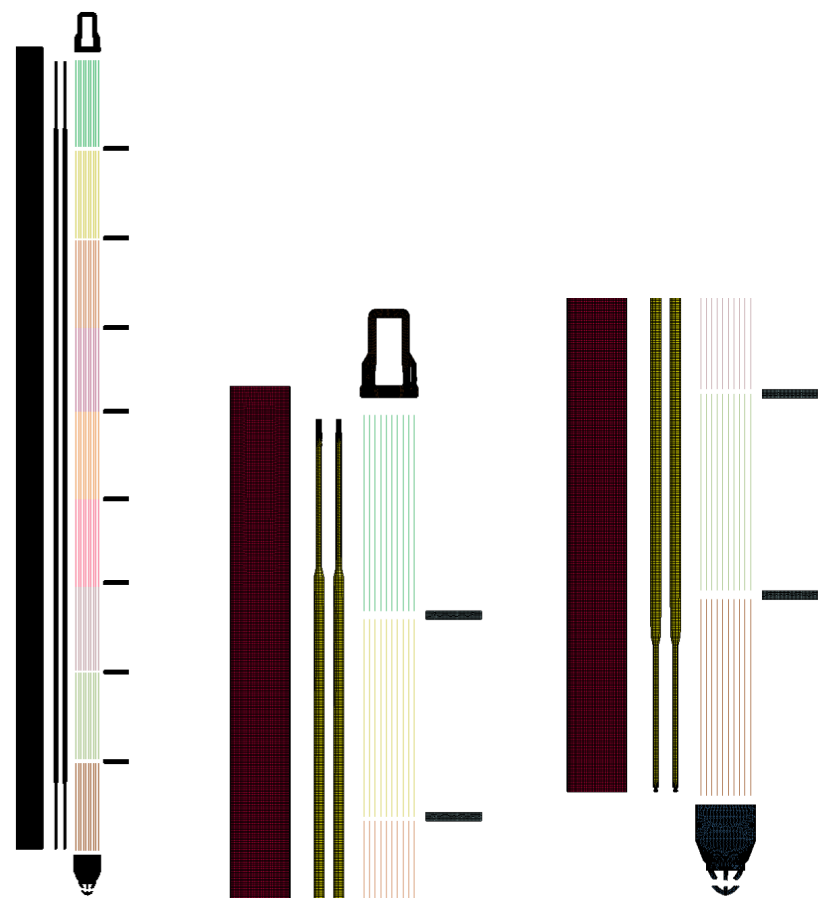

Fig. 13 Fuel assembly domain decomposition: all the substructures are represented as disconnected from the others.

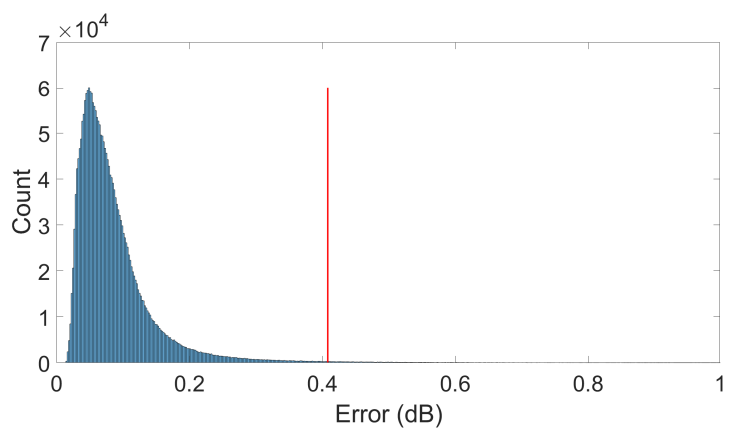

Fig. 14 Distribution of the error $\epsilon_{i j}$ for the multilevel CB model with $4 \mathrm{kHz}$ outer truncation and $6 \mathrm{kHz}$ inner truncation. Global error $\epsilon=\mu_{\epsilon}+4 \times \sigma_{\epsilon}=0.41 \mathrm{~dB}$ (red line).

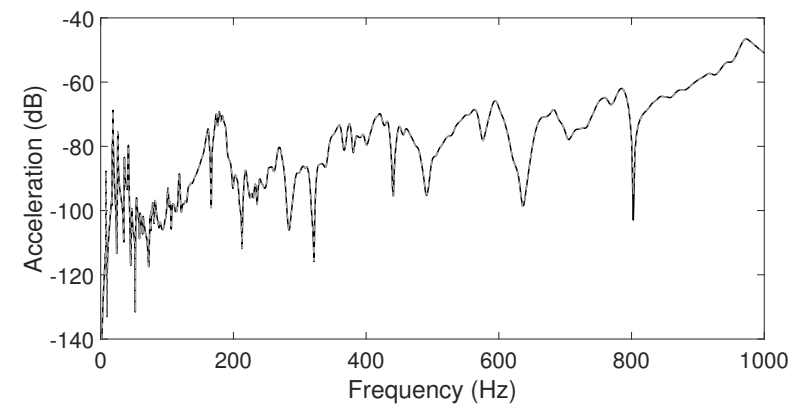

Fig. 15 FRF comparison between the $20 \mathrm{kHz}$ CraigBampton reference (black) and the multilevel Craig-Bampton model with $4 \mathrm{kHz}$ outer truncation and $6 \mathrm{kHz}$ inner truncation (dashed gray). Error: $\epsilon_{i j}=0.41 \mathrm{~dB}$. (one per FA). The reduced set of $N_{I}=63 \mathrm{DOF}$ of interest depicted in Fig. 7 as well as a slightly coarser frequency sampling are considered $\left(n_{\omega}=1000\right.$ instead of $n_{\omega}=3000$ frequency points). The importance value given by the error $\epsilon$ in decibel is obtained for all the $n_{A}=14,047 \mathrm{FA}$ modes below $4 \mathrm{kHz}$ of each of the 68 FA (these modes are approximated by the inner $\mathrm{CB}$ ). The FA modes are sorted (see Figs. 16 and 17) and a convergence analysis with respect to the percentage of FA modes retained is carried out (see Fig. 18). It can

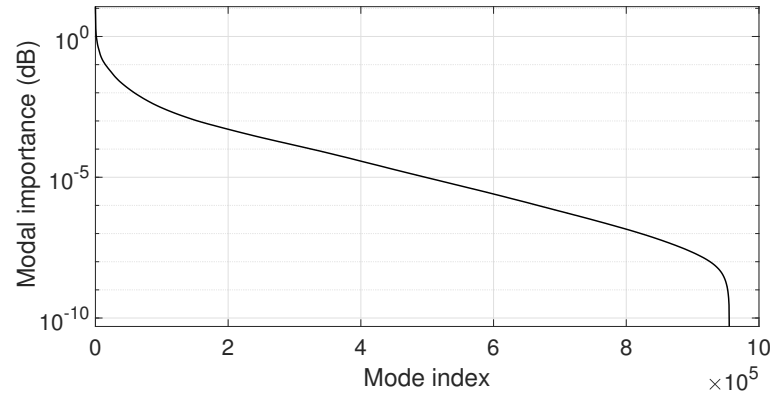

Fig. 16 Importance value $(\mathrm{dB})$ of all the fuel assembly modes (sorted).

be observed in Fig. 17 that the modal importance is not exactly the same from FA to FA. These differences can be explained by the different locations within the canister. It can be observed in Fig. 18 that about $75 \%$ of the FA modes do not contribute much to the vibration response. Keeping only the $25 \%$ most dominant FA modes within each FA, the error is very close to that of the multiscale modal analysis. For this choice of truncation $\left(n_{A}=3512\right)$, the distribution of the error is plotted in Fig. 19 and one example of FRF comparison that has the same error as the global error is presented

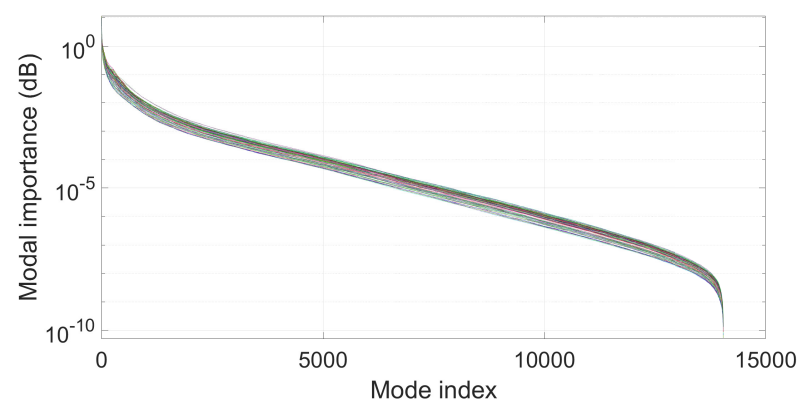

Fig. 17 For each fuel assembly, importance value (dB) of its modes (sorted).

in Fig. 20. As can be seen, the error level is as low as before and the accuracy of the FRF is retained. The $75 \%$ 


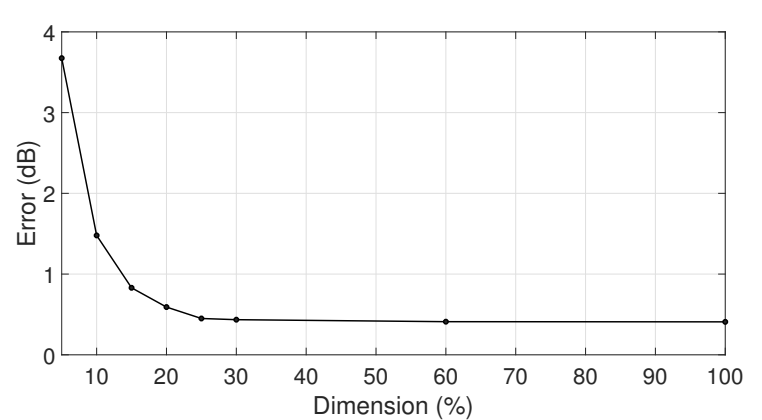

Fig. 18 Convergence of the global error $\epsilon=\mu_{\epsilon}+4 \times \sigma_{\epsilon}$ with respect to the percentage of dominant fuel assembly modes retained.

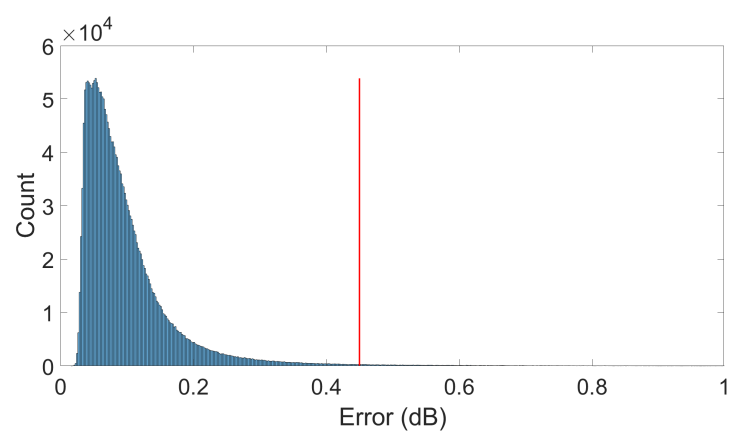

Fig. 19 Distribution of the error $\epsilon_{i j}$ for the proposed reduced-order model with $75 \%$ removal of the fuel assembly modes below $4 \mathrm{kHz}$ frequency. Global error $\epsilon=\mu_{\epsilon}+4 \times \sigma_{\epsilon}=$ $0.45 \mathrm{~dB}$.

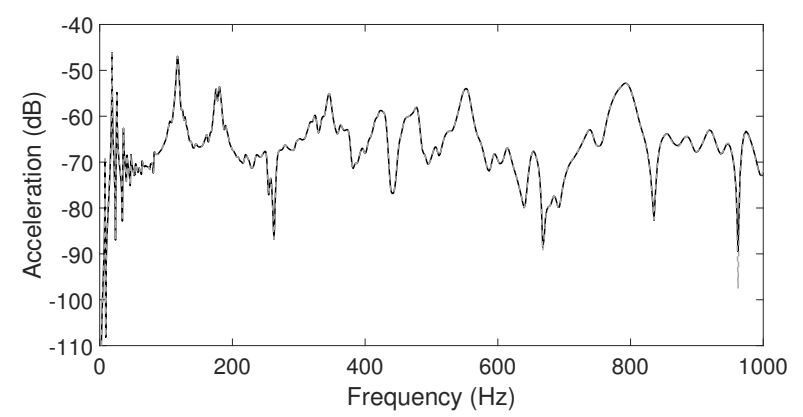

Fig. $20 \mathrm{FRF}$ comparison between the $20 \mathrm{kHz}$ CraigBampton reference (black) and the proposed reduced-order model with $75 \%$ removal of the fuel assembly modes below $4 \mathrm{kHz}$ frequency (dashed gray). Error: $\epsilon_{i j}=0.45 \mathrm{~dB}$.

FA mode removal allows the outer CB GEP of dimension $\nu=256,579$ (instead of $\nu=972,959$ ) to be solved more efficiently, in $\sim 21$ min with 68 nodes (instead of $118 \mathrm{~min})$. Taking into account the cost for determining the importance of each of the FA substructural modes, which took $\sim 14$ min with 68 nodes $(\sim 2 \mathrm{~min}$ for the reference FRF matrix and $\sim 12$ min for the $68 \times 14,047=955,196 \mathrm{FRF}$ matrices with FA mode removal), the proposed procedure allows a computational gain of a factor $\sim 3.5$ compared to the multiscale modal analysis presented in [14]. The modes that are obtained as eigenvectors of the outer CB GEP with FA mode removal are fewer but nonetheless they are able to preserve the same level of accuracy. The structural dynamics is accurately represented with only $n=232,586$ "modes" as substitutes of the usual $n_{e}=458,910$ modes associated with the FE approximation. This suggests that the usual modes do not necessarily constitute an adapted or optimal representation basis.

To highlight the importance of carefully evaluating the contribution of the substructural modes, Figure 21 shows the distribution of the error that is obtained when, instead of removing the $75 \%$ least dominant FA modes, only the one most dominant FA mode is removed. This single mode removal leads to an important error of $\epsilon=7.43 \mathrm{~dB}$. Figure 22 shows one example of FRF comparison exhibiting the same error: the single FA dominant mode removal is responsible for the large discrepancies in the high-frequency region of the whole system.

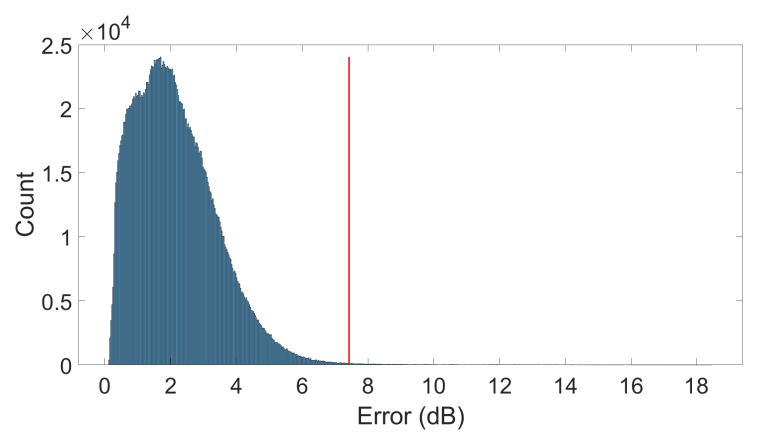

Fig. 21 Distribution of the error $\epsilon_{i j}$ for multiscale modal analysis with removal of the one most dominant fuel assembly mode. Global error $\epsilon=\mu_{\epsilon}+4 \times \sigma_{\epsilon}=7.43 \mathrm{~dB}$.

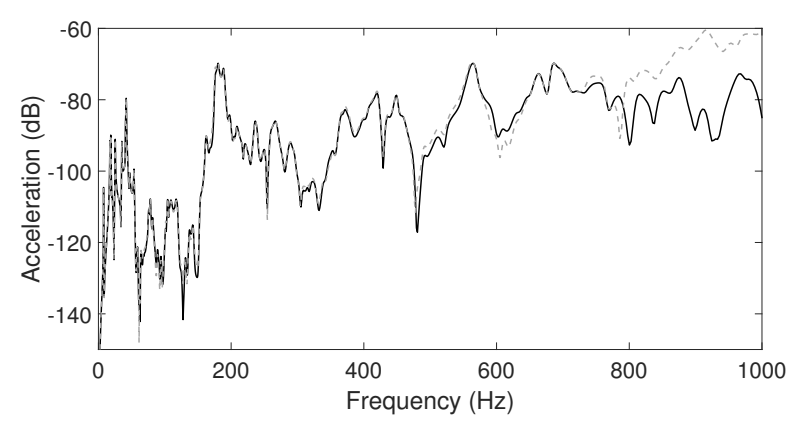

Fig. 22 FRF comparison between the $20 \mathrm{kHz}$ CraigBampton reference (black) and the multilevel Craig-Bampton model with $4 \mathrm{kHz}$ outer truncation and $6 \mathrm{kHz}$ inner truncation from which the one most dominant fuel assembly mode is removed (dashed gray). Error: $\epsilon_{i j}=7.43 \mathrm{~dB}$. 


\section{Conclusions}

A methodology for efficiently evaluating the importance of each of the substructural modes in a Craig-Bampton model has been proposed and validated for the case of a multilevel structure characterized by high modal density and localized structural connections. The importance of a substructural mode is given by the error induced by its sole removal and is calculated exactly for given frequency sampling and set of frequency response functions, which can both be adjusted with respect to efficiency. Through block factorization of the dynamic stiffness matrix using its Schur complement, the exact response matrix is efficiently calculated, from which the response matrix resulting from a single substructural mode removal can be readily obtained through a rank1 modification. For the fully-loaded spent nuclear fuel canister, the methodology allowed the removal of $75 \%$ of the substructural modes without significant loss of accuracy, which led to decrease the overall runtime by a factor of 3 to 4 .

Acknowledgements This research was supported by the United States Department of Energy through the Nuclear Energy University Program under the Contract No. DE-NE0008529. The findings presented herein are those of the authors and do not necessarily reflect the views of the sponsor.

\section{References}

1. Zienkiewicz OC, Taylor RL (2000) The finite element method. 5th ed., Butterworth-Heinemann, Oxford

2. Craig RR, Kurdila AJ (2006) Fundamentals of structural dynamics. Wiley, 2nd ed., John Wiley and Sons, Hoboken

3. Ohayon R, Soize C (2014) Advanced computational vibroacoustics - reduced-order models and uncertainty quantification. Cambridge University Press, New York

4. Lyon RH, DeJong RG (1995) Theory and Application of Statistical Energy Analysis. Butterworths-Heimann, Boston

5. Ericsson T, Ruhe A (1980) The spectral transformation Lanczos method for the numerical solution of large sparse generalized symmetric eigenvalue problems. Math Comput 35:1251-1268. https://doi.org/10.1090/S0025-57181980-0583502-2

6. Grimes R, Lewis J, Simon H (1994) A shifted block Lanczos algorithm for solving sparse symmetric generalized eigenproblems. SIAM J Matrix Anal Appl 15(1):228-272. https://doi.org/10.1137/S0895479888151111

7. Bathe KJ, Wilson EL (1973) Solution methods for eigenvalue problems in structural mechanics. Int J Numer Meth Eng 6(2):213-226. https://doi.org/10.1002/nme.1620060207

8. Bathe KJ (2013) The subspace iteration method - revisited. Comput Struct 126:177-183. https://doi.org/10.1016/j.compstruc.2012.06.002

9. Craig R, Bampton M (1968) Coupling of substructures for dynamic analyses. AIAA J 6(7):1313-1319. https://doi.org/10.2514/3.4741
10. De Klerk D, Rixen DJ, Voormeeren SN (2008) General framework for dynamic substructuring: history, review and classification of techniques. AIAA J 46(5):1169-1181. https://doi.org/10.2514/1.33274

11. Bennighof JK, Lehoucq RB (2004) An automated multilevel substructuring method for eigenspace computation in linear elastodynamics. SIAM J Sci Comput 25(6):2084-2016. http://dx.doi.org/10.1137/S1064827502400650

12. Ragnarsson $\mathrm{P}$, Van Gaal $\mathrm{T}$, Pluymers $\mathrm{B}$, Donders S, Vandepitte D, Desmet W (2011) Fast approximation of synthesized frequency response functions with automated multi-level substructuring (AMLS). Finite Elem Anal Des 47(2):195-199. https://doi.org/10.1016/j.finel.2010.08.009

13. Wilkinson JH (1965) The algebraic eigenvalue problem. Clarendon Clarendon Press, Oxford

14. Ezvan O, Zeng X, Ghanem R, Gencturk B (2020) Multiscale modal analysis of fully-loaded spent nuclear fuel canisters. Comput Methods Appl Mech Eng (accepted for publication)

15. Saad Y, Sosonkina M (1999) Distributed Schur complement techniques for general sparse linear systems. SIAM J Sci Comput 21(4):1337-1356. https://doi.org/10.1137/S1064827597328996

16. Zhang F (2005) The Schur complement and its applications. Springer, New York

17. Kammer DC, Triller MJ (1994) Ranking the dynamic importance of fixed interface modes using a generalization of effective mass. Int J Anal Exp Modal Anal 9(2):77-98.

18. Kammer DC, Triller MJ (1995) Selection of component modes for Craig-Bampton substructure representations. 36th Structures, Structural Dynamics and Materials Conference, New Orleans, USA. https://doi.org/10.2514/6.1995-1299

19. Barbone PE, Givoli D, Patlashenko I (2003) Optimal modal reduction of vibrating substructures. Int J Numer Methods Eng 57(3):341-369. https://doi.org/10.1002/nme.680

20. Givoli D, Barbone PE, Patlashenko I (2004) Which are the important modes of a subsystem? Int J Numer Methods Eng 59(12):1657-1678. https://doi.org/10.1002/nme.935

21. Liao BS, Bai Z, Gao W (2007) The important modes of subsystems: a moment-matching approach. Int J Numer Methods Eng 70(13):1581-1597. https://doi.org/10.1002/nme.1940

22. Tayeb S, Givoli D (2011) Optimal modal reduction of dynamic subsystems: extensions and improvements. Int $\mathrm{J}$ Numer Methods Eng 85(1):1-30. https://doi.org/10.1002/nme.2949

23. Palomba I, Richiedei D, Trevisani A (2015) Energy-based optimal ranking of the interior modes for reduced-order models under periodic excitation. Shock Vib Article ID 348106:1-10. https://doi.org/10.1155/2015/348106

24. Palomba I, Richiedei D, Trevisani A (2016) Mode selection for reduced order modeling of mechanical systems excited at resonance. Int J Mech Sci 114:268-276. https://doi.org/10.1016/j.ijmecsci.2016.05.026

25. Kim SM, Kim JG, Chae SW (2016) Evaluating mode selection methods for component mode synthesis. AIAA J 54:2852-2863. https://doi.org/10.2514/1.J054936

26. Kim SM, Kim JG, Park KC, Chae SW (2018) A component mode selection method based on a consistent perturbation expansion of interface displacement. Comput Methods Appl Mech Eng 330:578-597. https://doi.org/10.1016/j.cma.2017.11.010 
27. Lee J (2019) A parametric reduced-order model using substructural mode selections and interpolation. Comput Struct 212:199-214. https://doi.org/10.1016/j.compstruc.2018.10.018

28. Golub GH, Van Loan CF (1983) Matrix computations. John Hopkins Univ Press, Baltimore

29. Hestenes MR, Stiefel E (1952) Methods of conjugate gradients for solving linear systems. J Res Natl Bur Stand 49(6):409-436.

30. Parlett B (1980) The symmetric eigenvalue problem. Prentice Hall, Englewood Cliffs, NJ

31. Bathe KJ (2014) Finite element procedures. 2nd ed., Watertown, MA

32. Ezvan O, Zeng X, Ghanem R, Gencturk B (2019) Dominant vibration modes for broadband frequency analysis of multiscale structures with numerous local vibration modes. Int J Numer Methods Eng 117(6):644-692. https://doi.org/10.1002/nme.5971

33. Hallquist JO (2006) LS-DYNA theory manual. Livermore software Technology corporation 3:25-31.

34. MATLAB Release 2018b (2018) The Mathworks, Inc., Natick, Massachusetts, USA 